\title{
Application of higher order dynamic mode decomposition to modal analysis and prediction of power systems with renewable sources of energy
}

DOI:

10.1016/j.jjepes.2021.107925

\section{Document Version}

Accepted author manuscript

Link to publication record in Manchester Research Explorer

Citation for published version (APA):

Jones, C., \& Utyuzhnikov, S. (2022). Application of higher order dynamic mode decomposition to modal analysis and prediction of power systems with renewable sources of energy. International Journal of Electrical Power \& Energy Systems, 138(6), 1-14. https://doi.org/10.1016/j.ijepes.2021.107925

Published in:

International Journal of Electrical Power \& Energy Systems

\section{Citing this paper}

Please note that where the full-text provided on Manchester Research Explorer is the Author Accepted Manuscript or Proof version this may differ from the final Published version. If citing, it is advised that you check and use the publisher's definitive version.

\section{General rights}

Copyright and moral rights for the publications made accessible in the Research Explorer are retained by the authors and/or other copyright owners and it is a condition of accessing publications that users recognise and abide by the legal requirements associated with these rights.

\section{Takedown policy}

If you believe that this document breaches copyright please refer to the University of Manchester's Takedown Procedures [http://man.ac.uk/04Y6Bo] or contact uml.scholarlycommunications@manchester.ac.uk providing relevant details, so we can investigate your claim.

\section{OPEN ACCESS}




\title{
Application of higher order dynamic mode decomposition to modal analysis and prediction of power systems with renewable sources of energy ${ }^{\star}$
}

\author{
Jones, C. N. S. ${ }^{1}$, Utyuzhnikov, S.V. ${ }^{1,2}$ \\ ${ }^{1}$ Department of Mechanical, Aerospace \& Civil Engineering, \\ the University of Manchester, Manchester, M13 9PL, U.K. \\ ${ }^{2}$ Moscow Institute of Physics 83 Technology, Dolgoprudny 141700, Russia
}

\begin{abstract}
Concern for climate change is driving a vastly increased use of electricity and variable renewable energy supply encourages larger and evermore interconnected power systems. Stability analysis and short-term prediction of power system output has never been more urgent or more complex. Many distributed and renewable generators contribute zero inertia to the system and increase the risk of poorly damped oscillations leading to cascading outage. Datadriven techniques, higher order dynamic mode decomposition (HODMD) and total-least-squares higher-order dynamic mode decomposition (THDMD) are applied to modal analysis and short-term prediction of frequency and power exchange deviations. The decomposition uses multiple and randomized sampling windows of historical measurements. Dominant THDMD and HODMD modes can be used to show the contribution of renewable generation, such as
\end{abstract}

\footnotetext{
*This document is the result of the research project funded by the EPSRC (EP/L016141/1) through the Power Networks Centre for Doctoral Training.

Email address: s.utyuzhnikov@manchester.ac.uk (Jones, C. N. S. ${ }^{1}$, Utyuzhnikov, S.V. ${ }^{1,2}$ )
} 
wind power, to wide-area oscillations. The developed techniques are applied to the analysis of blackouts in Europe (2006) and the UK (2019), as well as the separation event in Australia (2018). The obtained results demonstrate that the damping of some HODMD modes can be overestimated. Although selected HODMD modes can reconstruct and predict power system output, the results are not always reliable. In turn, THDMD can predict dominant oscillations, with reduction of noise bias error in modal analysis of noisy measurements. With low noise data both techniques can produce very similar modal results.

Keywords: higher order dynamic mode decomposition, total-least-squares higher-order dynamic mode decomposition, power system stability analysis, oscillatory modes, wide-area monitoring, prediction

\section{Introduction}

International targets to reduce carbon emissions and to reduce the impact of climate change encourage a greater use of electrical power. Heating and transport, especially, are set to place unprecedented demands on power systems. Often great distances between suitable locations for renewable generation (such as wind farms, which are often remote) and the load, such as electric vehicle charging points, require a growing use of capacitance for voltage control. Increasing grid connection of renewable generators complicates frequency control, due to grid connection via power electronics with a reduced system inertia. Regional fossil-fuelled generators have for many decades provided a controllable power supply with physical electromechanical connection of the rotor speed to the grid frequency. 
Greater current drawn by a greater load, with many more heat pumps, is causing power systems to be operated closer to their voltage stability threshold [1]. Mixed requirements of the load for reactive power (with a combination of electronic devices, capacitive solar generation and inductive heat pumps), complicate the estimation of how close is a given system to a critical point for voltage instability.

Traditional equations of voltage and torque for modelling generation are suited only to synchronous machines and do not apply to renewable sources of energy. With up to six equations for the full model per generator, it is not possible to estimate system stability close to real time, with current computational power. Larger, highly interconnected systems increase the complexity of computation for stability analysis of power systems close to real time. Continental interconnected systems increase the urgency of online stability analysis and near-future prediction of the behaviour of a system. Renewable energy favours long-distance transmission to export power, for economic reasons and to make greatest use of all available carbon-neutral power.

If known, the system of equations governing a power system would be highly nonlinear, due to the interconnected nature of generators and load influencing rotor speeds and acceleration. Weather-related output of renewable generators is also nonlinear.

A data-driven approach in the form of dynamic mode decomposition (DMD) [2] is used in this work for stability analysis and near-future prediction of power system output, without the need for any governing equations. Using measured output of power systems prior to blackout, an exponential series 
is formed to approximate the nonlinear power system dynamics. This exponential series of complex conjugate eigenvalues is scaled by amplitudes and normalized modes, which represent the contribution of each location to each frequency of oscillation. Real parts of the eigenvalues represent the damping of modes and imaginary parts represent the frequencies. Nonlinear dynamics of the power system are thus approximated by linear dynamics, equivalent to the solutions of an unknown system of ordinary differential equations, which approximate the true system.

Susuki and Mezić pioneer the use of data-driven Koopman mode analysis for modal analysis of power system blackouts, using actual power system recordings in [3]. The Koopman analysis is closely related to the standard DMD, formulated such that the number of modes is determined by the number of samples. Modes are not selected for physical relevance, however. Barocio et al. apply standard DMD to power system modal analysis of PMU recordings in the review paper [4]. Whilst modal analysis is performed, neither full reconstruction nor prediction are performed, in [3] and [4].

Ramos et al. [5] use standard DMD to form a library of dominant modes, which are then selected via compressive sensing DMD. The accuracy of reconstruction, but not prediction, of a simulated voltage profile is assessed. Ramos et al. seek to identify optimally sparse sensor locations, although the sampling windows used for DMD are extremely short. The sampling window used is less than the wave period of the lower frequency inter-area oscillations, which can be $0.1 \mathrm{~Hz}$. This approach does not address noise, as it lacks redundancy of snapshots, and actual PMU measurements are not used. The simulated test system does not include renewable sources of energy. 
Dynamic mode decomposition in its original, numerically robust form [2] has the limitation that the number of modes can not be greater than the spatial dimension of the snapshot measurements. The series of modes and eigenvalues of power system output are exposed in this work, therefore, using variants of DMD. As introduced by Koopman [6] and as explained in [7], non-linear dynamics might have infinitely many eigenvalues and continuum spectrum.

In order to increase the number of available modes which can be calculated, Le Clainche et al. introduce a higher order dynamic mode decomposition (HODMD) in [8]. Higher order DMD is able to reveal a greater number of modes in a more accurate way than the standard DMD, as delay-coordinates of measured data are denoised and then stacked in matrix blocks. Le Clainche et al. [8] thus combine DMD, which globally identifies dynamics throughout a sampling window, with a moving-window technique based on Takens' delay-embedding theorem [9].

In HODMD, stacked measurements of a system are expressed in a reduceddimensional basis, prior to the modal analysis and computation of eigenvalues. The reduced-dimensional basis is found using the singular value decomposition (SVD) [8]. For denoising, the SVD is performed twice in HODMD. (Details of the SVD can be found in a linear algebra textbook such as [10]).

Le Clainche et al. [8], [11] discard the smaller singular values below a threshold, since these correspond to low-energy orthogonal components of the measurements. Small singular values are therefore regarded as noisy insignificant components of measured data [8]. Plots of relative error of reconstruction are used in [8] to estimate a threshold for truncation of singular 
values. Thus, low-dimensional bases of measurements of fluid flows are used within HODMD in existing studies of fluid dynamics [12], [11], and for prediction of turbulent flows [13], [14]. All samples are used in the computation of amplitudes.

Alassaf et al. in [15] employ the randomized DMD, developed in [16] for computationally efficient DMD in the presence of white noise. In [15], sequentially delayed and stacked measurements are oversampled on multiplication by a Gaussian random matrix. These data are used to form a reduceddimensional orthogonal basis, onto which the snapshots are projected.

As noted by Le Clainche et al. [8] and [13], DMD and HODMD modes which reconstruct data can be spurious artefacts of the sampling window. Le Clainche et al. [8] recommend that a range of singular value thresholds and stacking parameters be tested for consistently low reconstruction error, in order to identify physically meaningful modes. Alassaf et al. perform reconstruction within short sampling windows but not prediction. All identified modes are used. The technique of Alassaf et al. [15] reduces noise in PMU measurements and its computational efficiency is designed for analysis in real time. It does not, however, address bias error due to an imbalance in the treatment of noise in DMD, described by Dawson et al. [17].

Whilst the application of HODMD by Vega and Le Clainche [14] often concerns turbulent fluid flows with high process noise, Cheng et al. [18] focus on reduction of bias error due to additive white measurement noise. Cheng et al. devise the total-least-squares higher-order dynamic mode decomposition (THDMD) for the prediction of the remaining useful life of machinery using recordings of bearing vibration [18]. This THDMD is an adaptation 
to HODMD which projects stacked, delay-coordinate measurements onto a reduced-dimensional basis. Crucially, this basis is formed using two stacked sets of earlier and later measurements in [18], for a total-least-squares estimation of the system dynamics.

Since numerous modes are exposed by these higher order techniques, it is necessary to select modes suitable for prediction. Le Clainche et al. apply the mode selection energy criterion of Kou and Zhang [19] to the prediction of transient flows in [13]. In order to extract the relevant true dynamics, single-frequency modes are sorted in decreasing order of energy throughout the snapshot time series. The highest-energy modes are retained, which dominate the dynamics throughout the sampling window. Noise bias error is reduced using THDMD to reduce imbalance in the treatment of noise within HODMD.

This work seeks physically relevant dynamics: frequencies, damping and oscillatory modes must be physically meaningful for prediction and not spurious. Spurious modes can occur due to noise or aliasing within a given sampling window and some modes can be too well-damped to be useful for prediction. As noted in [8], physically relevant modes for prediction should be consistent for a range of values of $d$, the number of stacks of delay-coordinate snapshot data. Modes are selected for their influence throughout the sampling window.

This paper is organized as follows. The DMD and underlying Koopman operator theory, necessary for HODMD, are briefly introduced in Section 2. Then, this section introduces the higher order variant of DMD, HODMD, and the THDMD for reduction of error due to measurement noise. The 
two higher-order techniques, HODMD with energy criterion (HODMDc) and THDMD with energy criterion for selection of modes (THDMDc), are used in Section 3 for near-future prediction of power system measurements. Both techniques are used first to predict the South Australia frequency during the Queensland and South Australia separation event of 25th August 2018. Then, we consider blackouts in the UK of 9th August 2019 and Europe of 4th November 2006. This is followed by the Conclusion.

\section{Methodology}

\subsection{Introduction to the Dynamic Mode Decomposition}

Kutz et al. [20] provide an introduction to DMD [2] and to the underlying Koopman operator theory [6]. This theory is based on the Koopman linear operator matrix, $\mathcal{K}$, which acts upon a set of scalar-valued observation functions, $g$, of the system states, $\mathbf{x}_{k}$, in a time series of snapshot vectors at each time, $k: \mathbf{x}_{k} \in \mathbb{R}^{J}$. As is noted in [20], the DMD operator is a finitedimensional approximation to an infinite-dimensional Koopman operator. A snapshot, here, is a column vector of measurements of power system output, such as frequency, taken at various locations at the same time. The matrix of snapshots consists of a time series of such vectors, observed at a uniform sampling interval, $\Delta t$. The Koopman operator transforms a snapshot vector, $g\left(\mathbf{x}_{k}\right)$, into the observation at the next time step, $g\left(\mathbf{x}_{k+1}\right)$, one time step later:

$$
\mathcal{K} g\left(\mathbf{x}_{k}\right)=g\left(\mathbf{F}\left(\mathbf{x}_{k}\right)\right)=g\left(\mathbf{x}_{k+1}\right),
$$

where $\mathbf{F}$ represents the system transition. 
In DMD, using a finite sampling window and with a finite number of snapshots, the DMD operator, A, is used to approximate the Koopman operator. In matrix form, this system transition is the following:

$$
\mathbf{X}_{2}=\mathbf{A X}_{1}
$$

where $\mathbf{X}_{1}=\left[\mathbf{x}_{1}, \mathbf{x}_{2}, \ldots, \mathbf{x}_{K-1}\right], \mathbf{X}_{2}=\left[\mathbf{x}_{2}, \mathbf{x}_{3}, \ldots, \mathbf{x}_{K}\right], K$ is the number of snapshots. Thus, $\mathbf{X}_{2}$ represents a matrix of snapshot vectors one time step later than the snapshot columns of matrix $\mathbf{X}_{1}$. In this work, it is assumed that the measurements are equal to the state, such that $\mathbf{x}_{k}=g\left(\mathbf{x}_{k}\right)$, for $k=1,2, \ldots, K$.

Similarly, the DMD operator can be expressed as a locally linear approximation to the system dynamics [20]:

$$
\frac{d \mathbf{x}}{d t}=\mathbf{A} \mathbf{x} .
$$

In order to find operator $\mathbf{A}$ in Eq. (2), the standard DMD algorithm first decomposes the earlier set of snapshots via the SVD: $\mathbf{X}_{1}=\mathbf{U} \mathbf{\Sigma} \mathbf{V}^{*}$.

Here, $\mathbf{U} \in \mathbb{R}^{J \times R}$ and $\mathbf{V} \in \mathbb{R}^{K \times R}$ are complex unitary matrices; $\boldsymbol{\Sigma} \in$ $\mathbb{R}^{R \times R}$ is a pseudo-diagonal rectangular matrix with positive diagonal elements $\sigma_{k},(k=1, \ldots, R)$ called the singular values; the Hermitian, or complex conjugate, transpose is denoted by the asterisk. It is supposed that $\sigma_{k} \geq \sigma_{k+1}(k=1, \ldots, R-1)$.

In DMD, the earlier snapshot matrix, $\mathbf{X}_{1}$, is expressed in a reduceddimensional basis, using the dominant $r$ singular values and corresponding singular vectors. The use of SVD retaining only these $r$ largest singular values, $\boldsymbol{\Sigma}_{r}$, and their corresponding orthonormal singular vectors is referred as 
the truncated SVD. The minimum number, $r$, of singular values and corresponding singular vectors to be retained is determined by a threshold, $\epsilon_{S V D}$ [14]:

$$
\sqrt{\frac{\sigma_{r+1}^{2}+\cdots+\sigma_{R}^{2}}{\sigma_{1}^{2}+\cdots+\sigma_{R}^{2}}} \leq \epsilon_{S V D} .
$$

Alternative techniques for thresholding of singular values can be found in [20].

The DMD operator can then be found via the SVD-based pseudoinverse matrix $\mathbf{X}_{1}: \mathbf{A}=\mathbf{X}_{2} \mathbf{V}_{r} \boldsymbol{\Sigma}_{r}^{-1} \mathbf{U}_{r}^{*}$. Here, $\mathbf{U}_{r} \in \mathbb{R}^{J \times r}, \mathbf{V}_{r} \in \mathbb{R}^{K \times r}, \boldsymbol{\Sigma}_{r} \in \mathbb{R}^{r \times r}$, $\Sigma_{r}^{-1}$ is the pseudoinverse matrix to $\boldsymbol{\Sigma}_{r}$ with the inverse non-zero elements.

In the standard DMD [2], instead of $\mathbf{A}$, its low-dimensional projection is usually used:

$$
\breve{\mathbf{A}}=\mathbf{U}_{r}^{*} \mathbf{A} \mathbf{U}_{r}=\mathbf{U}_{r}^{*} \mathbf{X}_{2} \mathbf{V}_{r} \boldsymbol{\Sigma}_{r}^{-1}
$$

Next, consider the eigenvalues of the DMD operator in Eq. (5) which are the frequencies (imaginary parts) and damping values (real parts) of the power system oscillations, according to the eigenvector equation [20]: $\breve{\mathbf{A}} \mathbf{W}=\mathbf{W} \boldsymbol{\Lambda}$. The $r$ eigenvalues, $\lambda_{n}$ for $n=1 \ldots r$, are expanded in an exponential sequence of increasing powers. This expansion forms the time series trajectories, $\exp \left(\left(\delta_{n}+i \omega_{n}\right)(k-1) \Delta t\right)$, where $\ln \left(\lambda_{n}\right) / \Delta t=\delta_{n}+i \omega_{n}$ for snapshot numbers $k=1, \ldots, K[8]$.

Normalized modes, $\breve{\phi}_{n}$, the spatial contributions of each measurement location to the oscillations, multiply this eigenvalue expansion, together with the modal amplitudes, $a_{n}$. As shown by Tu et al. [21] and discussed in [20], the exact DMD modes, $\breve{\Phi}$ are calculated as follows:

$$
\breve{\boldsymbol{\Phi}}=\mathbf{X}_{2} \mathbf{V}_{r} \boldsymbol{\Sigma}_{r}^{-1} \mathbf{W}
$$


The number of exponential terms in this series, $r$, is the spectral complexity of the decomposition [14]. Thus, each later snapshot vector is decomposed as follows:

$$
g\left(\mathbf{x}_{k+1}\right)=\mathcal{K} g\left(\mathbf{x}_{k}\right) \simeq \breve{\mathbf{A}} g\left(\mathbf{x}_{k}\right)=\sum_{n=1}^{r} a_{n} \breve{\phi}_{n} \exp \left(\left(\delta_{n}+i \omega_{n}\right) k \Delta t\right)
$$

where $k=1, \ldots, K$ are the snapshot column numbers.

\subsection{Higher order dynamic mode decomposition}

Higher order DMD [8] is a variant of DMD, to expose a broader spectrum of modes than the modes available via standard DMD. This may be necessary, especially with noisy snapshot data, to improve the linear approximation to the dynamics of a nonlinear system [8].

Higher order dynamic mode decomposition uses the full snapshot matrix, denoted $\mathbf{X}$. An initial denoising step uses the truncated SVD with a singular value threshold $\epsilon_{S V D}$, as in Eq. (4). The SVD threshold, $\epsilon_{S V D}$, is set according to an approximate point of change in the trend of singular

values, as in [8]. Denoised, so-called reduced snapshots, $\hat{\mathbf{X}}$, are formed by reconstruction of the $r$ retained singular values and temporal right singular vectors: $\hat{\mathbf{X}}=\Sigma_{r} \mathbf{V}_{r}^{*}$.

Higher order DMD relates the reduced snapshots in a sliding-window system transition, using the higher-order Koopman assumption [8]:

$$
\hat{\mathbf{x}}_{k+d} \simeq \hat{\mathbf{A}}_{1} \hat{\mathbf{x}}_{k}+\hat{\mathbf{A}}_{2} \hat{\mathbf{x}}_{k+1}+\cdots+\hat{\mathbf{A}}_{d} \hat{\mathbf{x}}_{k+d-1}, \text { for } k=1, \ldots, K-d
$$

where $d>1$ is the order. Higher order DMD can also be referred as DMD-d. 
Enlarged-reduced, modified snapshots, $\tilde{\mathbf{x}}_{k}$, are formed and the transition of Eq. (8) is: $\tilde{\mathbf{x}}_{k+1} \simeq \tilde{\mathbf{A}} \tilde{\mathbf{x}}_{k}, \quad k=1, \ldots, K-d$, where:

$$
\tilde{\mathbf{x}}_{k}=\left[\begin{array}{c}
\hat{\mathbf{x}}_{k} \\
\hat{\mathbf{x}}_{k+1} \\
\vdots \\
\hat{\mathbf{x}}_{k+d-1}
\end{array}\right], \quad \tilde{\mathbf{A}}=\left[\begin{array}{ccccc}
\mathbf{0} & \mathbf{I} & \mathbf{0} & \ldots & \mathbf{0} \\
\mathbf{0} & \mathbf{0} & \mathbf{I} & \ldots & \mathbf{0} \\
\vdots & \vdots & \vdots & \ddots & \vdots \\
\mathbf{0} & \mathbf{0} & \mathbf{0} & \ldots & \mathbf{I} \\
\hat{\mathbf{A}}_{1} & \hat{\mathbf{A}}_{2} & \hat{\mathbf{A}}_{3} & \ldots & \hat{\mathbf{A}}_{d}
\end{array}\right] .
$$

The modified snapshot matrix is $\tilde{\mathbf{X}} \in \mathbb{R}^{d r \times K-d+1}$. Modified Koopman matrix, $\tilde{\mathbf{A}}$, is the higher-order counterpart of the standard DMD operator, $\breve{\mathbf{A}}$, in Eq. (7).

Since the modified Koopman matrix is a $(d r \times d r)$-matrix and is usually noisy and high-dimensional, the truncated SVD is used a second time, similarly to Eq. (4), upon $\tilde{\mathbf{X}}$. The number of retained singular values is denoted $M$, which is the spectral complexity of the higher-order decomposition. The matrices of $M$ left singular vectors, singular values and right singular vectors retained at this stage are denoted $\tilde{\mathbf{U}}_{M}, \tilde{\boldsymbol{\Sigma}}_{M}$ and $\tilde{\mathbf{V}}_{M}$, respectively.

The reduced-enlarged-reduced snapshots, produced using this second truncated SVD, form a $(M \times K-d+1)$-matrix, $\check{\mathbf{X}}=\tilde{\mathbf{\Sigma}}_{M} \tilde{\mathbf{V}}_{M}^{*}$. The earliest $K-d$ reduced-enlarged-reduced snapshots in $\check{\mathbf{X}}$ are denoted $\check{\mathbf{X}}_{1}$ and those one time step later are denoted $\check{\mathbf{X}}_{2}$. The pseudoinverse, $\check{\mathbf{X}}_{1}^{\dagger}=\check{\mathbf{V}}_{1} \check{\check{\Sigma}}_{1}^{-1} \check{\mathbf{U}}_{1}^{*}$, provides a least-squares solution $\check{\mathrm{A}}=\check{\check{X}}_{2} \check{\check{X}}_{1}^{\dagger}$, which minimizes the $l_{2}$-norm || $\check{\mathbf{A}}_{1}-\check{\mathbf{X}}_{2} \|_{2}$. The HODMD eigenvalues, denoted $\check{\check{\lambda}}_{m}, m=1,2, \ldots, M$, are the eigenvalues of $\check{\text { Ă. }}$

Eigenvectors of $\check{\check{A}}$ are left-multiplied by the left singular vector matrix, $\tilde{\mathbf{U}}_{M}$. The high-dimensional eigenvectors are then reduced, by retaining only 
the last $r$ elements of each eigenvector, and normalized to form the reduced modes, $\check{\check{\phi}}_{m}$. Amplitudes, $a_{m}$, are formed by the solution of a linear system, to minimize the error of HODMD reconstruction of all snapshots.

The matrix of $r$-dimensional reduced modes, $\check{\check{\Phi}}$, is scaled by the amplitudes and left-multiplied by the spatial left singular vectors, $\mathbf{U}_{r}$ (formed using the first truncated SVD). The modes are finally normalized to have RMS $=1$, to form the matrix of $M$ HODMD modes, $\Phi \in \mathbb{R}^{J \times M}$. Amplitudes, $a_{m}$, are transformed, such that they are real and positive.

The original snapshots, $\mathbf{x}_{k}$, for $k=1,2, \ldots, K$, are approximated using the HODMD expansion:

$$
\mathbf{x}_{k} \simeq \sum_{m=1}^{M} a_{m} \phi_{m} \check{\check{\lambda}}_{m}^{k-1}=\sum_{m=1}^{M} a_{m} \phi_{m} \exp \left(\left(\check{\check{\delta}}_{m}+i \check{\omega}_{m}\right)(k-1) \Delta t\right),
$$

where $\check{\check{\delta}}_{m}+i \check{\omega}_{m}=\frac{1}{\Delta t} \ln \left(\check{\check{\lambda}}_{m}\right)$ and $\Delta t$ is the snapshot sampling interval.

\subsection{Total-least-squares higher-order dynamic mode decomposition}

Some noise almost inevitably remains in snapshots when the HODMD operator is computed, despite the use of truncated SVD for denoising. This is often evident in a curved and unclear change in behaviour of singular values, when RRMSE of truncated SVD reconstruction is plotted for a range of $r$ retained singular values, using Eq. (4). The setting of $\epsilon_{S V D}$ as an estimation of the noise threshold is often unclear, therefore, as $\epsilon_{S V D}$ set too high would curtail the basis for genuine dynamics [8], [11].

Total-least-squares higher-order dynamic mode decomposition (THDMD) was devised by Cheng et al. in [18] to adapt HODMD for analysis of remaining useful life of machinery, with high measurement noise of bearing 
oscillations. This adaptation employs theory of reducing bias error in the computation of the DMD operator, identified by Dawson et al. [17]. The HODMD operator is a least squares solution of the equation:

$$
\check{\check{A}}_{1}=\check{\check{X}}_{2}
$$

where $\check{\mathbf{X}}_{1}$ and $\check{\mathbf{X}}_{2}$ are the earlier and later reduced-enlarged-reduced snapshot matrices, respectively. In HODMD, this linear system is solved using the SVD-based pseudoinverse of the first $K-d$ reduced-enlarged-reduced snapshots, $\check{\check{\mathbf{X}}}_{1}=\check{\check{\mathbf{U}}}_{1} \check{\check{\Sigma}}_{1} \check{\check{\mathbf{V}}}_{1}^{*}$. The reduced-enlarged-reduced Koopman operator is thus produced:

$$
\check{\check{\mathbf{A}}}=\check{\check{\mathbf{X}}}_{2} \check{\mathbf{X}}_{1}^{*}\left(\check{\mathbf{X}}_{1} \check{\mathbf{X}}_{1}^{*}\right)^{\dagger},
$$

using an identity for the pseudoinverse $\left(\check{\mathbf{X}}_{1}\right)^{\dagger}$.

To expose the treatment of noise, $\check{\mathbf{X}}_{1}=\overline{\mathbf{X}}_{1}+\mathbf{N}_{X 1}$ and $\check{\mathbf{X}}_{2}=\overline{\mathbf{X}}_{2}+\mathbf{N}_{X 2}$ are substituted into Eq. (11). That is, where $\mathbf{N}_{X 1}$ and $\mathbf{N}_{X 2}$ represent the assumed zero-mean Gaussian sensor noise remaining in $\check{\check{\mathbf{X}}}_{1}$ and $\check{\check{\mathbf{X}}}_{2} ; \overline{\mathbf{X}}_{1}$ and $\overline{\mathbf{X}}_{2}$ are the reduced-enlarged-reduced snapshot matrices without measurement noise [17]. Only the even-valued powers, and not the odd-valued powers, of the noise terms can have non-zero expectation. Higher-order noise terms of the resulting infinite series are discarded, which gives the approximate expectation of the HODMD operator [17]:

$$
\mathbb{E}(\check{\check{\mathbf{A}}}) \simeq \check{\mathbf{A}}_{\text {true }}\left(I-\mathbb{E}\left(\mathbf{N}_{X 1} \mathbf{N}_{X 1}^{*}\right)\left(\overline{\mathbf{X}}_{1} \overline{\mathbf{X}}_{1}^{*}\right)^{-1}\right),
$$

where $\check{\check{A}}_{\text {true }}$ is the true HODMD operator, formed without the noise terms, $\mathbb{E}$ is the mathematical expectation operator.

Only the noise term, $\mathbf{N}_{X 1} \mathbf{N}_{X 1}^{*}$, of the earlier reduced-enlarged-reduced snapshot matrix, $\check{\check{X}}_{1}$, is considered in Eq. (12). Noise of the later set of 
snapshots, $\check{\mathbf{X}}_{2}$, is excluded. As explained in [17], this causes bias in the computation of the DMD operator and can result in the estimation of modes to be stable when they could be truly poorly damped. Inspired by the debiasing work of Dawson et al. [17] and Hemati et al. [22], Cheng et al. introduce THDMD [18].

Following the first truncated SVD in Eq. (4), an augmented snapshot array, $\mathbf{Z}$, is formed of the modified (stacked) snapshot matrices for THDMD: $\mathbf{Z}=\left[\begin{array}{ll}\tilde{\mathbf{X}}_{1}^{T} & \tilde{\mathbf{X}}_{2}^{T}\end{array}\right]^{T}$. Each of the earlier and later modified snapshot matrices are projected onto a reduced basis: $\mathbb{P}_{Z}=\mathbf{V}_{\rho} \mathbf{V}_{\rho}^{*}$, where $\mathbf{V}_{\rho}$ are the first $\rho$ right singular vectors of the augmented matrix. Similarly to the TDMD approach of Hemati et al. [22], $\rho$, the number of retained right singular vectors of $\mathbf{Z}$, does not exceed the number of rows of the modified snapshot matrix, $\tilde{\mathbf{X}}$. For the de-biasing projection step of THDMD, therefore, $\epsilon_{\mathbb{P}}$ is set as a threshold for the RRMSE of SVD reconstruction of modified snapshot matrix, $\tilde{\mathbf{X}}$. Similarly to Eq. (4), the singular values of $\tilde{\mathbf{X}}$ thus determine $\rho$. Thus, $\overline{\mathbf{X}}_{1}=\tilde{\mathbf{X}}_{1} \mathbb{P}_{Z}$ and $\overline{\mathbf{X}}_{2}=\tilde{\mathbf{X}}_{2} \mathbb{P}_{Z}$, where $\overline{\mathbf{X}}_{1}$ and $\overline{\mathbf{X}}_{2}$ represent the projected modified snapshot matrices [18].

The projected modified snapshot matrices are used in THDMD in place of the HODMD modified snapshot matrices. The remaining steps of THDMD continue as for HODMD, as described in [18]. It is expected that noise bias inherent in HODMD is to be minimized via THDMD, assuming that the measurement error follows a Gaussian distribution. It is proposed that the true nature of any unstable modes is therefore to be predicted using THDMD. 


\subsection{Mode selection and application of techniques}

Higher order variants of DMD expose a broad discrete spectrum of modal frequencies. In the HODMD algorithm [8], the spectral complexity of the decomposition, that is, the number of modes, equals the number of retained singular values. The SVD threshold, $\epsilon_{S V D}$, and stacking parameter, $d$, are calibrated using the RRMSE of HODMD reconstruction, similarly performed in [13]. Le Clainche et al. recommend selection of the SVD threshold close to a change in behaviour of the singular values in [8]. Using noisy data, the 'elbow' point in the RRMSE of SVD reconstruction values is often unclear and is used as a guide for a suitable $\epsilon_{S V D}$.

Unlike the high-dimensional fluid snapshots used in previous applications of HODMD, such as [13], the snapshots of available power system measurements have low spatial dimension. To maintain a spectral complexity high enough to expose modes suitable for prediction, $\epsilon_{S V D}$ must be low enough to maintain a suitable number of singular values and modes. The power system measurements must be denoised using a high enough setting of $\epsilon_{S V D}$, however, so that modes may be extrapolated for prediction.

Most of the modes are usually noisy. The advantage of the higher-order techniques over standard DMD, however, is that there is a greater probability that some of the many computed modes produce a good linear approximation to the true dynamics of the sampling interval. Test cases in this work are all power system disturbance events and the sampling intervals are all taken prior to, or during, a blackout. Transient modes seem to dominate during these times and so this work uses the energy criterion of Kou and Zhang [19] for selection of modes. 
This energy criterion is applied to transient fluid flow around wind turbines in [13]. As explained in [19] and [13], normalized modes $\phi_{m}$ are ranked and sorted in descending order of energy index, $I_{m}$. The energy index is the summation, an approximate integral, of the absolute value of each mode's time-varying coefficient throughout the interval of $K$ snapshots. Thus, the energy index is defined, based on [19], [13]:

$$
I_{m}=\sum_{k=1}^{K}\left|a_{m} \exp \left(\left(\check{\delta}_{m}+i \check{\tilde{\omega}}_{m}\right)(k-1) \Delta t\right)\right| \Delta t,
$$

where $a_{m}$ represents the amplitude and $\check{\check{\delta}}_{m}+i \check{\tilde{\omega}}_{m}=\ln \left(\check{\check{\lambda}}_{m}\right) / \Delta t$. The uniform snapshot sampling interval is $\Delta t$, and $\check{\check{\delta}}_{m}$ and $\check{\omega}_{m}$ represent the damping and angular frequency, respectively, of an $m$ th modal oscillation. A maximum of twenty complex conjugate eigenvalue pairs are retained each time when HODMDc or THDMDc is performed, in this work, according to the energy criterion.

Centering of snapshots data is shown to be beneficial in applications of DMD in [23], so the time series mean is subtracted from snapshots. Real eigenvalues (without oscillation) are therefore discarded as noise.

Since the recorded data of power output and frequency are noisy, it is important to test consistency of HODMD results, as described by Le Clainche et al. in [8] and by Vega et al. in [14]. Since a suitable sampling window is uncertain, and since the phase angles of the modal oscillations are unknown, a random variable sampling window is used in each blackout case. Three hundred random and shifted sampling windows of snapshots are decomposed via HODMDc and THDMDc for each test case. The resulting modes are stored. Inspired by the work of Hua et al. [24] for selection of consistent 
eigenvalues central to a neighbourhood, we fuzzy-cluster stored eigenvalues.

To ensure symmetry, such that retained modes and eigenvalues form complex conjugate pairs, clustering is performed using only the part of each eigenvalue conjugate pair with positive imaginary part. Once the eigenvalues with positive imaginary part and their corresponding modes have been selected, their complex conjugates are retained from the stored decomposition results.

Mode-pairs are tested for accuracy of conjugate pairs. Only mode-pairs with absolute difference less than $10^{-10}$ are retained. Complex-valued modes are rounded to eight decimal places. Inspired by Hua et al. [24], modes are then selected by the phase angles of their spatial elements. The argument vectors of modes corresponding to the eigenvalues in each cluster are then fitted to a multivariate Gaussian mixture model (GMM), for selection of the mode closest to the principal mean. Hua et al. [24] select a corresponding mode for each eigenvalue by minimizing the change in phase angle between neighbouring modes, produced using randomly shifted sampling windows. The multivariate GMM similarly allows for noise and for a slight phase shift due to the randomized snapshot windows.

The mode closest (by Euclidean norm) to the principal mean is selected, together with its corresponding eigenvalue. This clustering and selection of the eigenvalue-mode pairs is performed 300 times, due to slight uncertainty of optimization and of the cluster membership. Results are stored and the modes to be used for prediction are selected for lowest RRMSE of reconstruction.

The maximum reliable frequency recommended in [14] using HODMD 


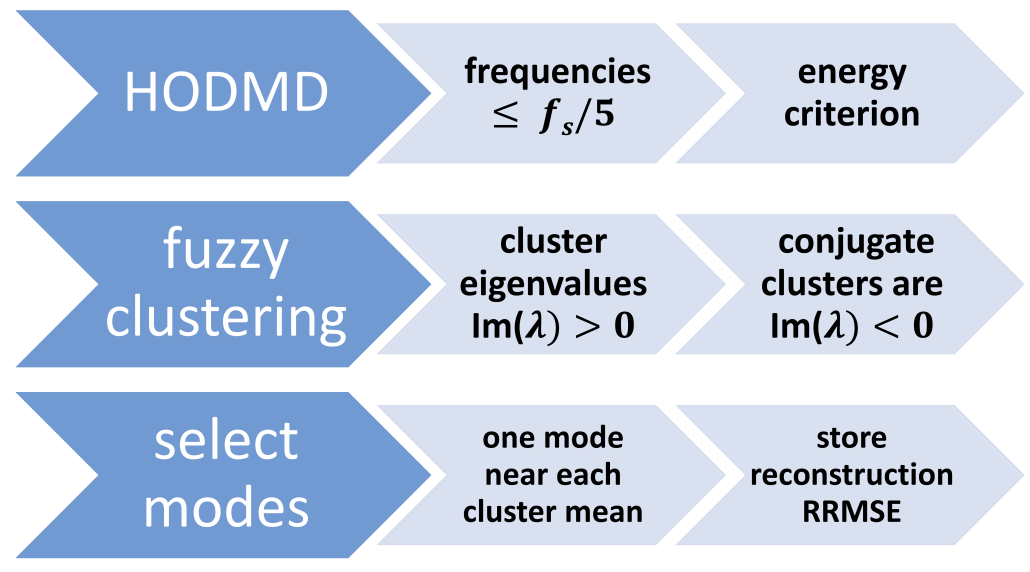

Figure 1: Summary of the process of HODMDc/THDMDc mode selection applied.

is approximately one fifth of the sampling frequency. If information of the original sampling frequency is not available, the approximate frequency of data obtained from plots via UN-SCAN-IT [25] is used. Modes oscillating at a frequency higher than one fifth of this approximate sampling frequency are discarded. The process of mode selection is summarised in the schematic diagram Figure 1.

The MATLAB code written by Le Clainche and Vega to accompany the text [14] is downloaded from Mendeley Data [26]. The published code for DMD-d, especially code for Chapter 2 of [14], is adapted and used throughout this work. Adaptations are especially made for THDMD and for alternative techniques for selection of modes.

\section{Near-future prediction of power system states}

3.1. Analysis of the Queensland and South Australia separation event, 2018 Snapshots are obtained from the Australian Energy Market Operator (AEMO) Final Report for the Queensland (QLD) and South Australia (SA) 
system separation on 25th August 2018 [27]. South Australia separated from Victoria at 13:11:46.8 h, due to emergency opening of the Heywood interconnector. This caused SA frequency to rise, as power could no longer be exported to Victoria. Snapshots are a time series of recorded SA frequency, power output measurements of six individual SA synchronous generators, SA wind power, SA battery power, SA solar power and SA synchronous power generated. Torrens Island B1 (TI B1) is one of the synchronous generators.

Snapshot data are obtained using UN-SCAN-IT graph scanning software [25] at a sampling frequency of approximately $1 \mathrm{~Hz}$. To ensure uniform sampling frequency, snapshot data are interpolated using inbuilt MATLAB function interp1.m. Linear interpolation, with extrapolation to endpoints, gives a time series of sampling interval $1 \mathrm{~s}$. These data are pre-processed using a Savitzky-Golay filter of order five. Snapshot data is thus used in a sampling interval of approximately 300 s starting from 13:25:00 h, with start and end times each plus or minus a random shift of up to $20 \mathrm{~s}$. The maximum frequency of retained modes is $0.2 \mathrm{~Hz}$, according to the recommendation of Vega et al. in [14].

Table A.1 in Appendix A shows the RRMSE of reconstruction values for HODMD using 300 s mean-subtracted snapshots during the SA separation event. For the setting $d=30$, singular values show changes of behaviour of RRMSE of SVD reconstruction close to $\epsilon_{S V D}=10^{-2}$ and $\epsilon_{S V D}=5 \times 10^{-4}$, which guides the range of $\epsilon_{S V D}$ to be tested for calibration. All Tables in Appendix A are produced with use of the MATLAB code of [28]. Table A.1 reveals the low reconstruction RRMSE value of 0.0658 for HODMD with SVD threshold 0.005 and parameter $d=30$. The SVD threshold $\epsilon_{S V D}=0.005$ 
is one of the highest settings tested to provide an RRMSE of reconstruction less than 0.1. For calibration purposes, all available HODMD modes are used with relative amplitude greater than the threshold value $\epsilon_{S V D}$.

Reconstruction RRMSE values for approximately $300 \mathrm{~s}$ are less than 0.1 for $d$ between 25 and 35 with $\epsilon_{S V D}=0.005$. For the de-biasing projection step of THDMDc, a lower SVD threshold is set as $\epsilon_{\mathbb{P}}=0.001$. These low RRMSE of reconstruction values show consistency, which satisfies the low sensitivity to $d$ recommended in [8]. The selected parameter settings $d=30$ and $d=35$, used for prediction, are close to one tenth of the 301 snapshots, as suggested in [14].

Similarly to Hua et al. [24] for use of consistent eigenvalues for prediction, the HODMDc/THDMDc eigenvalues of positive imaginary part are divided into 25 clusters using MATLAB [29] fuzzy clustering function fcm.m. To allow a small number of eigenvalues to belong to more than one cluster, the exponent of the fuzzy partition matrix is set at 3 .

In order to select one eigenvalue-mode pair for each cluster, modes corresponding to each cluster are fitted to a multivariate GMM of eleven dimensions (equal to the spatial dimension of the eleven-dimensional snapshots). The MATLAB function evalclusters.m is first used to estimate the number of GMM components, each component being a cluster of modes of similar phase angle which correspond to a given cluster of eigenvalues. The MATLAB function fitgmdist.m is then used to fit the mode angles to an eleven-dimensional GMM. A single Gaussian mixture component is identified. A regularization parameter 0.01 is used with fitgmdist.m for HODMDc and THDMDc with $d=30$, due to ill-conditioning. No regularization is 
necessary with $d=35$.

\subsection{1. $H O D M D c$ and $T H D M D c$ results}

Table A.2 reveals the RRMSE of HODMDc prediction of mean-subtracted snapshots using a maximum of 20 modes. This table of results is produced using a single snapshot window of $300 \mathrm{~s}$. Settings $d=30$ and $d=35$ with $\epsilon_{S V D}=0.005$ for HODMDc provide $300 \mathrm{~s}$ prediction with relatively low RRMSE values 6.802 and 3.145, respectively. With $d=35$, RRMSE of mean-subtracted prediction increases sevenfold to 22.517 for the slightly lower threshold $\epsilon_{S V D}=0.003$ and is as high as 98.507 for $\epsilon_{S V D}=0.01$. Some parameter settings of $d$ and $\epsilon_{S V D}$ can give deceptively low RRMSE of HODMDc prediction values due to very large, noisy oscillations about the mean. One such example is HODMDc with $d=50$ and $\epsilon_{S V D}=0.005$, which gives RRMSE of prediction of just 1.312 despite excessive exponential growth of predicted oscillations.

Reconstruction of SA frequency for $300 \mathrm{~s}$ and prediction for a further 300 $\mathrm{s}$ using five HODMDc modes is shown in Figure 2a. Using $\epsilon_{S V D}=0.005$, HODMDc prediction is closer to the original values with $d=35$ than it is with $d=30$. The same parameter settings are used for THDMDc, with $\epsilon_{\mathbb{P}}=$ 0.001 in Figure 2b. Five THDMDc modes provide close reconstruction and predicted oscillations peak close to the measured SA frequency. Prediction of the full original snapshots using the selected five HODMDc modes with $d=30$ has RRMSE $=0.0859$ and with $d=35$, prediction has RRMSE $=$ 0.0858. Prediction of the full original snapshot values using the selected five THDMDc modes, with $d=30$, has RRMSE equal to 0.0875 . With $d=35$, the RRMSE of THDMDc prediction is 0.0865 . 


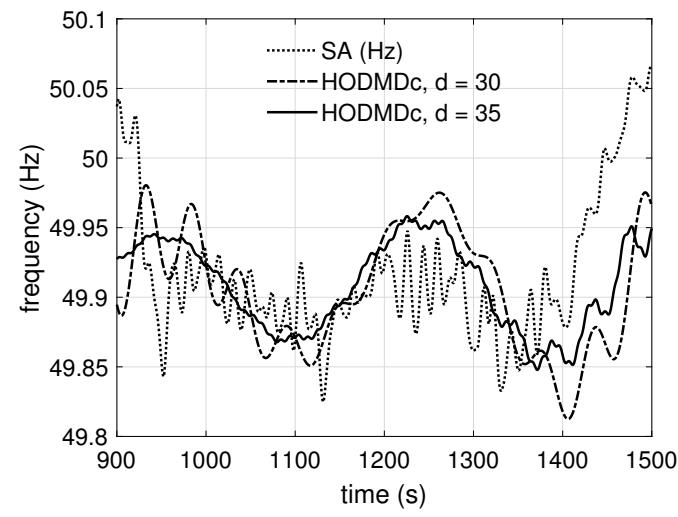

(a)

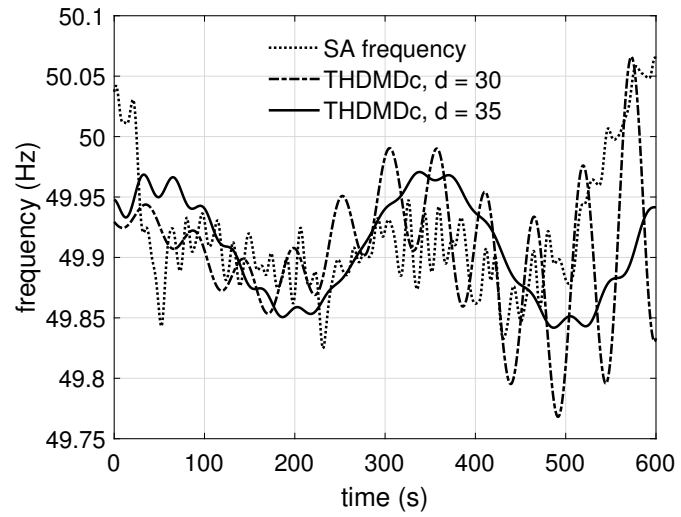

(b)

Figure 2: Reconstruction for $300 \mathrm{~s}$ from 13:25:00 $\mathrm{h}$ and $300 \mathrm{~s}$ prediction of SA frequency via (a) five HODMDc modes and (b) five THDMDc modes.

Reconstruction and prediction results with the same value $\epsilon_{S V D}=0.005$ but the higher value $d=35$ appear more robust to noise in Figures 2a and 2b. Modal oscillations are moderate and approximate the original measurements. The final increase in SA frequency is not accurately predicted and is largely underestimated, however, by both HODMDc and THDMDc after time 450 S.

Relative RMS error of 300 s prediction is shown using up to 25 HODMDc modes in Figure 3a and up to 25 THDMDc modes in Figure 3b. Results are produced using $d=35$ with $\epsilon_{S V D}=0.005$ and, for THDMDc, $\epsilon_{\mathbb{P}}=0.001$. Whiskers represent values which differ from the quartiles by 1.5 times the interquartile range in all box plots, throughout this work. All box plots are produced using supplementary code from [30]. Figures 3a and 3b reveal that median and quartiles of the clustering results give a low RRMSE of prediction, with median RRMSE values less than 0.09 for all HODMDc and 


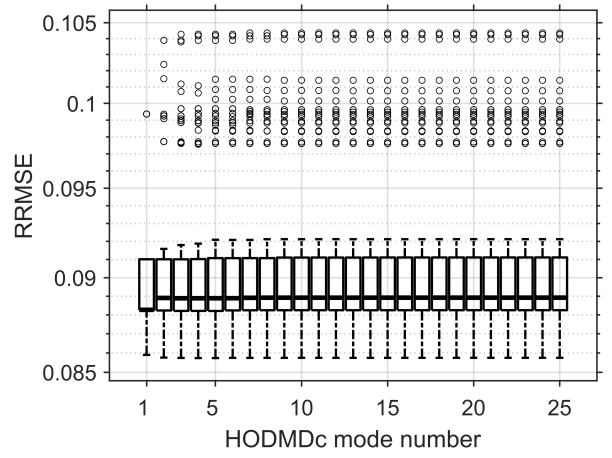

(a)

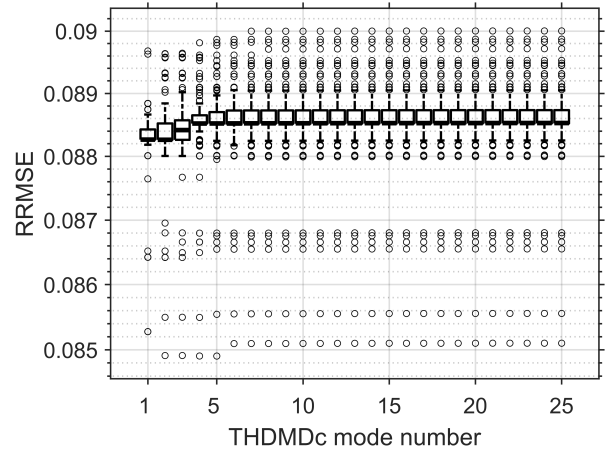

(b)

Figure 3: Box plots of RRMSE of prediction for 300 iterations of mode selection via clustering of (a) HODMDc modes and (b) THDMDc modes. Median lines are thickened and circles represent outliers.

THDMDc modes. The interquartile range of RRMSE for modal clustering results is higher for HODMDc, however, at approximately 0.003, as opposed to approximately 0.001 for THDMDc.

The box and whiskers plot in Figure 4a shows the initial reduction in median HODMDc modal energy index, from $I_{m}=92$ for mode one to $I_{m}=16$ for mode five. Figure $4 \mathrm{~b}$ shows a more gradual decline in median energy index of the dominant THDMDc modes, from $I_{m}=83$ for mode one to $I_{m}=16$ for mode eight. Higher modes, up to mode 20, have near-constant energy index of around ten for both HODMDc and THDMDc.

Similarly to the HODMDc analysis of Le Clainche et al. in [13], absolute damping values and amplitudes of modes are shown in Figures 5a and $5 \mathrm{~b}$ respectively. Results for modes which give the lowest RRMSE of reconstruction of all clustering results using 25 modes with $d=35$ are used here. Figure 5a shows that most of the HODMDc and THDMDc modes have a 


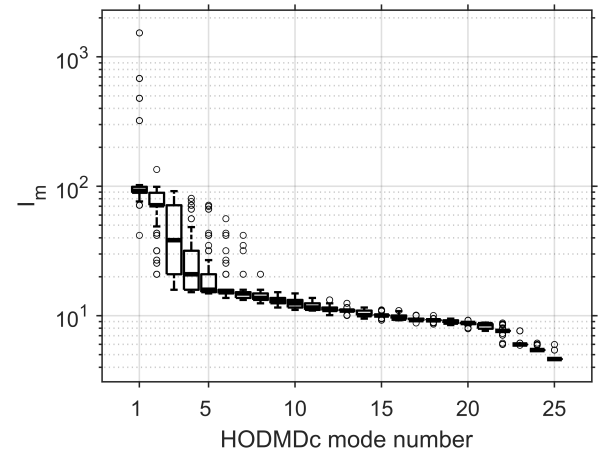

(a)

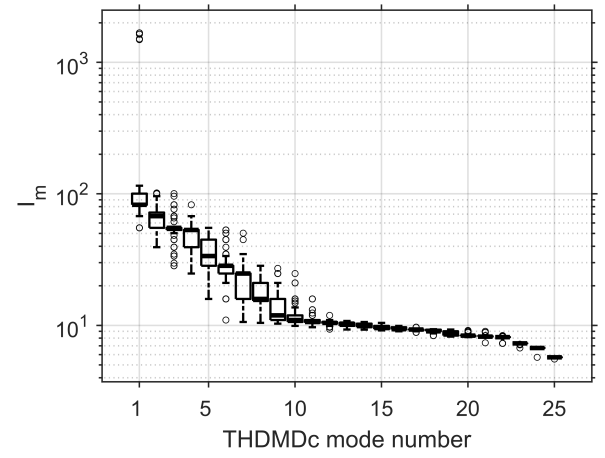

(b)

Figure 4: Box plots of modal energy indices, $I_{m}$, of results using 300 iterations of mode selection via clustering of (a) HODMDc modes and (b) THDMDc modes. Median lines are thickened and circles represent outliers.

small absolute damping value, less than 0.01 , and so could be classed as permanent modes. Several THDMDc modes of $|\delta|$ close to 0.01 have higher absolute damping values than the HODMDc modes. The transient nature of modes can therefore be stronger with THDMDc than HODMDc modes.

For both techniques, Figure 5b shows that one mode has amplitude close to an order of magnitude higher than the mode of second highest amplitude. Most of the modes lie in a band of amplitudes less than 0.1. Only three HODMDc modes have amplitude greater than 0.1 but six THDMDc modal amplitudes lie above 0.1 .

Compass plots in Figures $6 \mathrm{a}$ and $6 \mathrm{~b}$ show three dominant components of the HODMDc and THDMDc first modes with $d=35$, respectively. Each first mode is selected for lowest RRMSE of reconstruction. These plotted elements correspond to the SA wind power; the most dominant individual synchronous generator, TI B1, and an average of all SA synchronous generation. This 


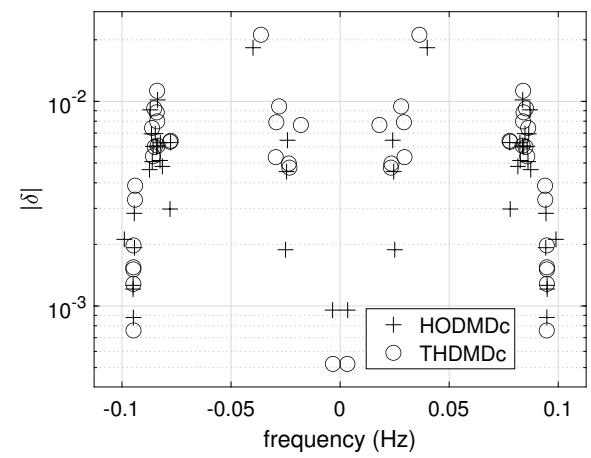

(a)

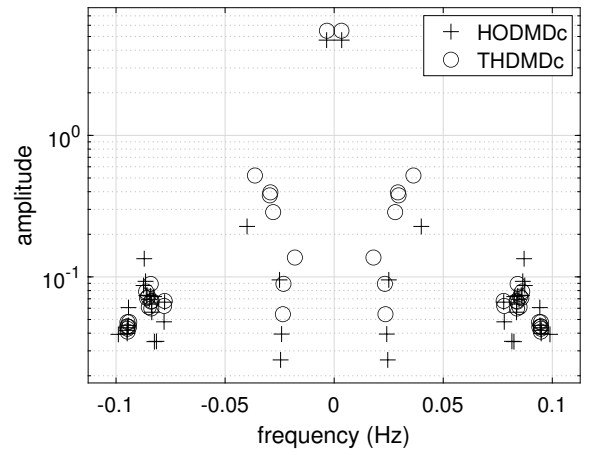

(b)

Figure 5: (a) Absolute damping and (b) amplitudes of 25 HODMDc and THDMDc modes.

polar form reveals the dominance of SA wind power following the islanding operation. Mode one of the HODMDc results shows strong agreement with THDMDc mode one, as approximate complex conjugates of the same mode pair.

Reconstruction and prediction of snapshots by extrapolation of modes for a further $300 \mathrm{~s}$ has low RRMSE using HODMDc and THDMDc. Most of the 25 selected modes have amplitude in the band of low amplitude values less than 0.1. Many modes have small absolute damping value and are permanent, however. Some THDMDc modes have higher amplitudes and absolute damping values than some of the HODMDc modes. Modal clustering results show very similar RRMSE of prediction overall, with lower interquartile range of RRMSE for the THDMDc than the HODMDc results. It is not possible using these HODMDc or THDMDc modes to accurately predict the sharp increase in SA frequency during the final $100 \mathrm{~s}$.

The influence of renewable energy in power system behaviour is identified by HODMDc and THDMDc. This is revealed by the strong participation of 


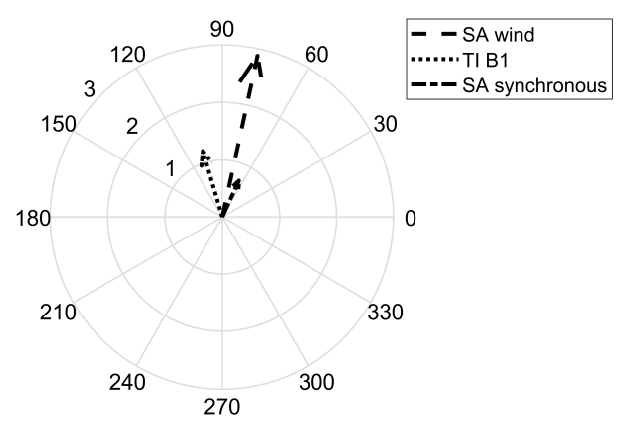

(a)

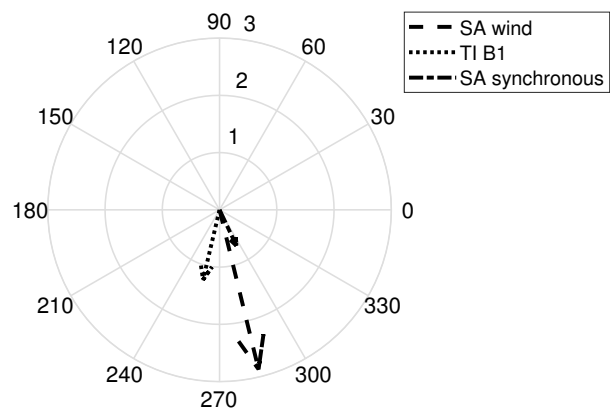

(b)

Figure 6: Dominant components SA wind power, TI B1 and SA synchronous generation of (a) HODMDc mode one and of (b) THDMDc mode one.

SA wind power in the most dominant HODMDc and THDMDc modes.

\subsection{Prediction of frequency, UK event of 9th August 2019}

On 9th August 2019, a fault occurred on the Eaton Socon-Wymondley circuit at 16:52:33.490 h, according to the National Grid ESO (NGESO) Technical Report [31]. Three lightning strikes were recorded close to the Eaton Socon-Wymondley circuit at 15:52:33 h. Although the fault cleared within approximately $70 \mathrm{~ms}$, Hornsea wind farm lost $737 \mathrm{MW}$ and CCGT units tripped at Little Barford in a cascade of events. At 16:53:49.398 h, grid frequency fell below $48.8 \mathrm{~Hz}$, triggering low frequency demand disconnection [31]. To maintain the steady-state $50.0 \mathrm{~Hz}$ frequency, NGESO had secured a $1000 \mathrm{MW}$ loss of power infeed at 16:52:26 h [31].

Snapshot data are obtained from National Grid ESO records of historical frequency data [32]. Frequency data have uniform $1 \mathrm{~Hz}$ sampling frequency. The maximum frequency of retained modes is therefore $0.2 \mathrm{~Hz}$. Higher order 
DMD with energy criterion and THDMDc are applied to approximately 400 $\mathrm{s}$ snapshots in the minutes before the blackout in Subsection 3.2.1. Snapshot windows have pseudorandom uniformly distributed start times from 16:43:00 h plus or minus $20 \mathrm{~s}$. Snapshot window duration is similarly pseudorandom, at $400 \mathrm{~s}$ plus or minus $20 \mathrm{~s}$.

The RRMSE of SVD reconstruction values for a range of retained singular values are shown in Figure $7 \mathrm{a}$ for $d=180$. Values close to changes in trend in the RRMSE shown in Figure 7a are tested for a range of $d$, for calibration of the SVD threshold. Table A.3 shows the RRMSE of HODMD reconstruction values using $400 \mathrm{~s}$ mean-subtracted snapshots from 16:43:00 h. The singular value tolerance of $\epsilon_{S V D}=0.004$, used for this UK case for HODMDc and THDMDc, is set at a point close to a change in behaviour of the RRMSE of SVD reconstruction. Setting $\epsilon_{S V D}=0.004$, with $d=180$, is also the highest SVD threshold tested to give an RRMSE of HODMD reconstruction less than 0.1. Using these settings, HODMD reconstruction has RRMSE $=$ 0.067 and the RRMSE is consistently low (less than 0.2) for $d$ between 160 and 190. For THDMDc, a lower singular value threshold of $\epsilon_{\mathbb{P}}=0.003$ is set for the initial projection and truncation of right singular values.

Use of all available HODMD modes, of the 401 snapshots, with $d=40$ produces very large amplitude oscillations, without denoising. A similar application of HODMD with the higher value $d=180$ reduces the frequency of noisy oscillations but reconstruction fails due to oscillations of very large amplitude. Figure $7 \mathrm{~b}$ shows the failed reconstruction and prediction of UK frequency using all HODMD modes and retaining all singular values.

Eigenvalues produced using HODMDc/THDMDc are formed into 20 clus- 


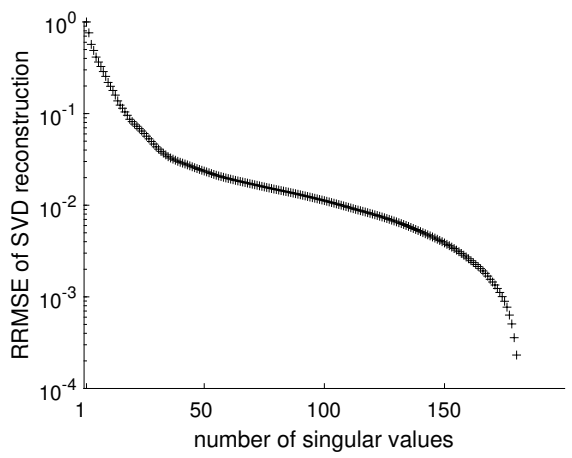

(a)

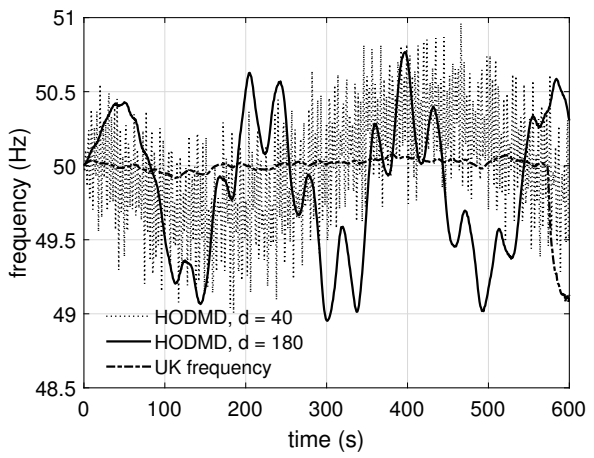

(b)

Figure 7: (a) Relative RMS error of SVD reconstruction with $d=180$ and (b) HODMD reconstruction for $400 \mathrm{~s}$ from 16:43:00 $\mathrm{h}$ and prediction of $200 \mathrm{~s}$ with $d=40$ and $d=180$, without denoising.

ters, using the MATLAB fuzzy clustering function fcm.m. The exponent of the fuzzy partition matrix is 3 for MATLAB function fcm.m. Modes are fitted to a single Gaussian distribution using MATLAB function fitgmdist.m, with zero regularization. Using the selected eigenvalue-mode pairs, reconstruction of snapshots is performed for $400 \mathrm{~s}$ from 16:43:00 $\mathrm{h}$ and prediction is tested for a further $200 \mathrm{~s}$.

3.2.1. HODMDc and THDMDc results for the 9th August 2019 UK blackout

The RRMSE of prediction results are very low, less than 0.01, using up to 20 HODMDc modes in Figure 8a or 20 THDMDc modes, as shown in Figure $8 \mathrm{~b}$, for analysis of the UK frequency.

Energy indices decline gradually on a logarithmic scale. Median energy indices decrease from 10.3 and 8.5 for HODMDc and THDMDc modes one, respectively, to less than 0.1 for both HODMDc and THDMDc modes 20 in respective Figures 9a and 9b. Mode two has higher median energy index 


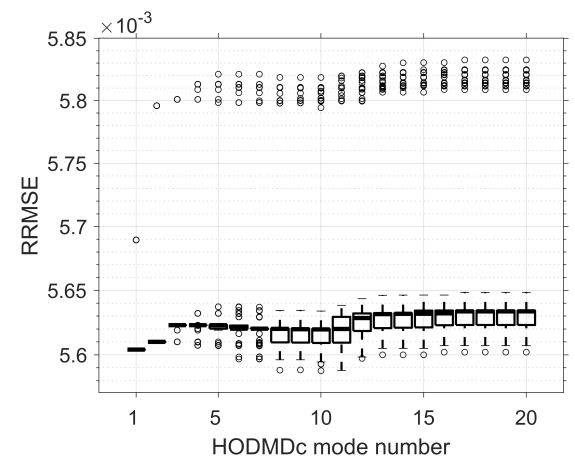

(a)

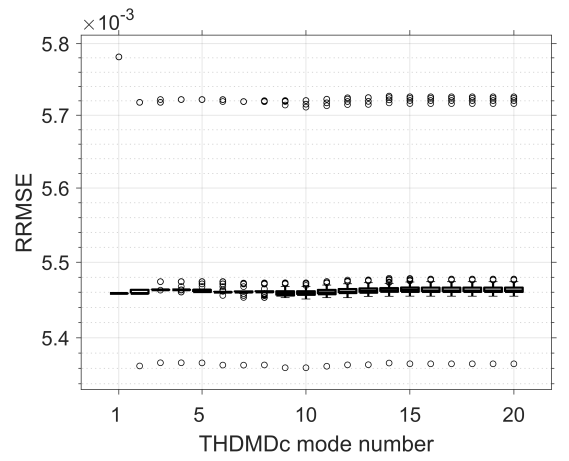

(b)

Figure 8: Box and whiskers plots of RRMSE of prediction using 20 modes via (a) HODMDc and (b) THDMDc. Median (thickened) and quartiles are shown for 300 iterations of mode selection. Circles represent outliers.

using THDMDc, at $I_{m}=8.5$, compared to $I_{m}=4.8$ for HODMDc mode two. Interquartile range of all energy indices is low, with few outliers.

Several HODMDc modes and THDMDc modes in Figure 10a are seen to be transient, with high absolute damping values greater than 0.01 . One THDMDc mode has the highest absolute damping value, greater than 0.1. In Figure 10b, almost all modes have low amplitude less than 0.1. Most modal amplitudes lie above $10^{-3}$ and one THDMDc mode has the highest amplitude. Modes produced via THDMDc and HODMDc have similarly negative correlation of frequency and amplitude. The 20 eigenvalues of the clustering results with lowest RRMSE of reconstruction, with $d=180$ and $\epsilon_{S V D}=0.004$, are shown.

Prediction results from time $400 \mathrm{~s}$ to $600 \mathrm{~s}$, using five HODMDc and five THDMDc modes are shown in Figures 11a and 11b, respectively. Relative RMS error of prediction of full values using the selected five HODMDc modes 


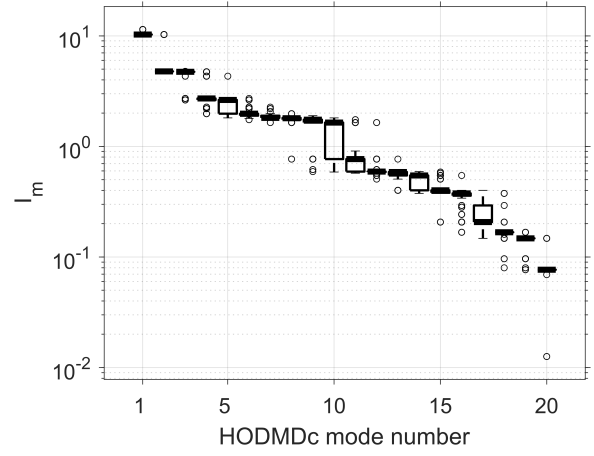

(a)

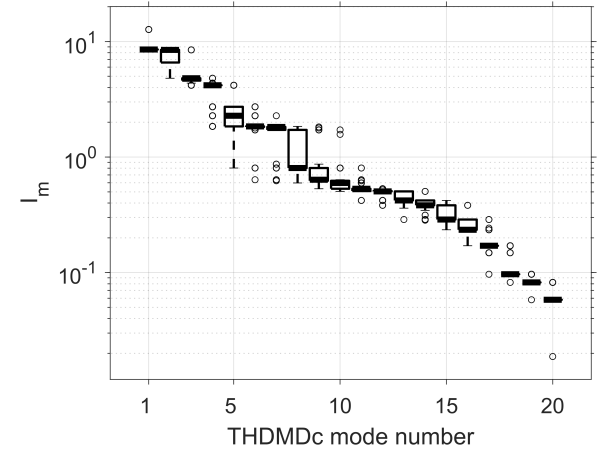

(b)

Figure 9: Box and whiskers plots of energy index, $I_{m}$, of 20 modes of UK frequency via (a) HODMDc and (b) THDMDc. Median (thickened) and quartiles are shown for 300 iterations of mode selection. Circles represent outliers.

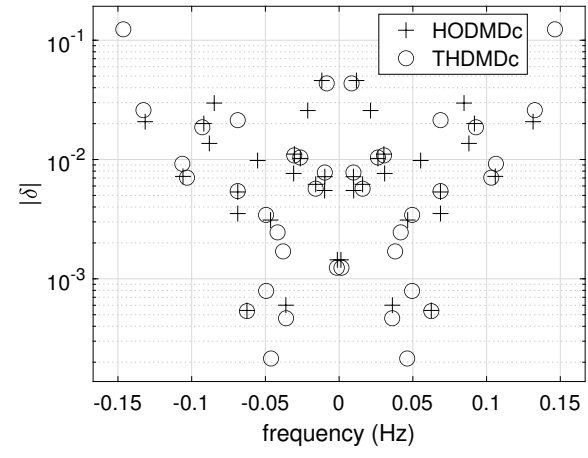

(a)

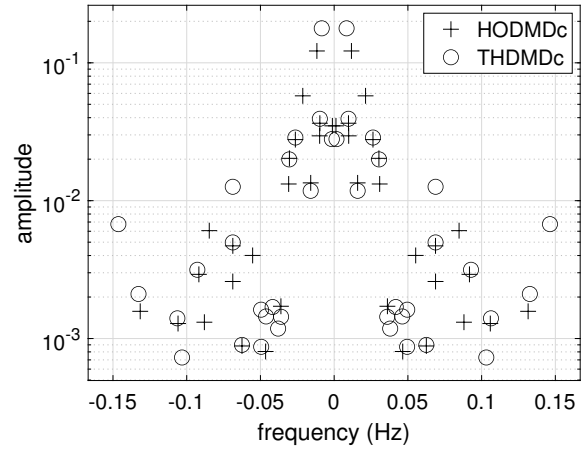

(b)

Figure 10: (a) Absolute damping and (b) amplitudes of 20 HODMDc and THDMDc modes of UK frequency. 


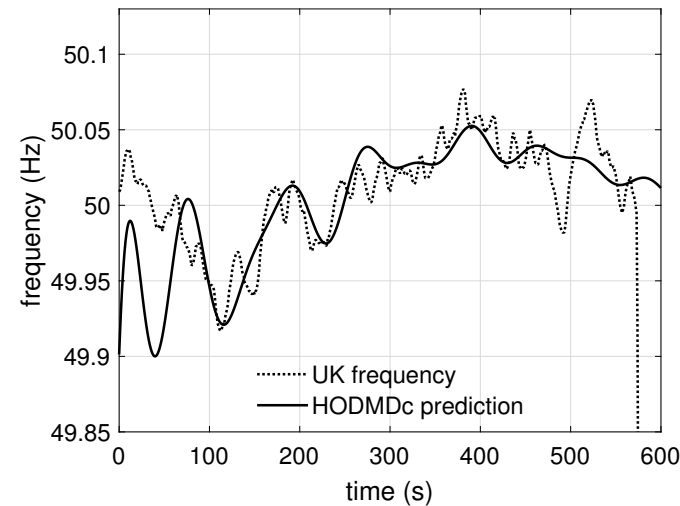

(a)

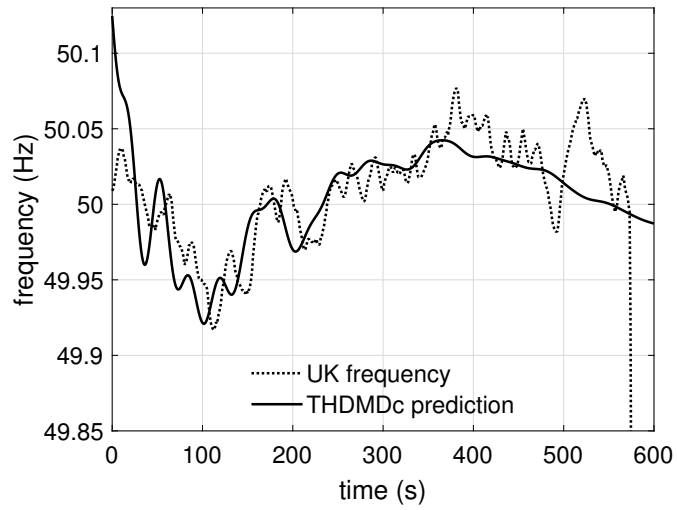

(b)

Figure 11: Reconstruction of UK frequency for $400 \mathrm{~s}$ from 16:43:00 $\mathrm{h}$ and prediction of a further $200 \mathrm{~s}$ using (a) five HODMDc modes and (b) five THDMDc modes.

is $5.64 \times 10^{-3}$, compared to $5.46 \times 10^{-3}$ using the selected five THDMDc modes. The sharp decline in frequency following the circuit fault at Eaton Socon-Wymondley, after 574 s, could not be predicted using HODMDc or THDMDc.

In this UK test case, both HODMDc modes and THDMDc modes are able to approximate the oscillations of frequency during the initial $400 \mathrm{~s}$ sampling time. A small number, such as five, dominant modes can approximate the oscillations. Modes computed via THDMDc provide greater detail of early reconstruction around the time $100 \mathrm{~s}$. A relatively high value of $d=180$, close to half of the number of snapshots, provides a high degree of delayembedding, without excessive loss of influence of the later snapshots. The moderately high setting of $d$ also gives a high level of redundancy of singular values and modes for selection, which improves the accuracy of reconstruction and prediction. Prediction results are similar, lying close to a moving average 
for both techniques, although THDMDc prediction has slightly lower RRMSE than HODMDc prediction. Neither HODMDc nor THDMDc can predict the cascading drop in UK frequency towards the blackout, following the circuit fault at 16:52:33.490 h.

\subsection{Analysis of European power exchange deviations, 2006}

Higher order DMD with energy criterion and THDMDc are applied to the European blackout of 4 th November 2006. To enable the passage of a ship on the Ems River, the two circuits of the Conneforde-Diele line in Germany were disconnected at 21:38 $\mathrm{h}$ and 21:39 $\mathrm{h}$. The disconnection took place earlier than originally planned. Telephone calls between operators E.ON Netz, RWE TSO and Vattenfall Europe Transmission between 21:46 h and 21:52 $\mathrm{h}$ expressed concern that the system was operating close to overload and close to thresholds of protection settings. The rushed coupling of two busbars at 22:10 h, without due calculation, led to a cascading outage and around 15 million European customers lost power supply.

Snapshot data are obtained from plots in the Union for the Co-ordination of Transmission of Electricity (UCTE) Final Report (2007) [33] via UNSCAN-IT graph scanning software [25]. As recommended by Vega et al. [14], the maximum retained frequency of eigenvalues is $0.2 \mathrm{~Hz}$ here. Data are obtained from the plots in [33] at slightly unequal intervals between approximately $1 \mathrm{~s}$ and $2 \mathrm{~s}$. The original sampling frequency is not disclosed in the Final Report. Data from the plots are linearly interpolated to form 1201 uniformly sampled snapshots of sampling interval $1 \mathrm{~s}$. The data are

pre-processed to reduce noise via a MATLAB Savitzky-Golay filter of order five. 
In order to expose early instability, eight European power exchange deviations (power exchange minus scheduled power exchange [33]), denoted PEDs, are studied after 21:50 h for approximately $300 \mathrm{~s}$. Snapshot sampling windows each start at a pseudorandom time selected from a uniform distribution from 21:50:20 h, plus or minus up to $20 \mathrm{~s}$. Higher order DMD with energy criterion and THDMDc are each performed using randomized sampling windows, of $300 \mathrm{~s}$ plus or minus up to $20 \mathrm{~s}$. Higher order DMD with energy criterion and THDMDc techniques are applied for short-term prediction of PEDs, by extrapolation of the calculated modes for a further $150 \mathrm{~s}$.

Table A.4 shows RRMSE of reconstruction values for HODMD using $300 \mathrm{~s}$ of snapshots from 21:50:20 h. Table A.4 reveals the low RRMSE of reconstruction (of mean-subtracted values), at 0.0723 with $\epsilon_{S V D}=0.002$ and $d=35$. Low RRMSE values, less than 0.2 , are also recorded between $d=25$ and $d=40$, which shows consistency. Lower SVD threshold values offer little reduction in RRMSE, so the relatively high threshold $\epsilon_{S V D}=0.002$ is selected for denoising.

Both HODMDc and THDMDc use a singular value truncation threshold of $\epsilon_{S V D}=0.002$. The additional singular value threshold of $\epsilon_{\mathbb{P}}=0.001$ is used for the THDMD denoising step, in which snapshots are projected onto a correlation matrix of truncated right singular vectors.

Eigenvalues are stored, from the 300 randomized sampling windows, and are formed into fifteen clusters for consistency, using the MATLAB fuzzy clustering function fcm.m. To allow a small number of eigenvalues to have membership of more than one cluster, an exponent 3 is used for the fuzzy partition matrix for fcm.m. Modes corresponding to the eigenvalues in each 
cluster are then fitted to an eight-dimensional Gaussian model using the MATLAB function fitgmdist.m with zero regularization. MATLAB function evalclusters.m is used to determine the number of mean values and components of the GMM. A single Gaussian mixture component is identified.

\subsubsection{HODMDc and THDMDc results for the European blackout, 2006}

Results in Figures 12a and 12b are produced using the fifteen eigenvalues selected for lowest RRMSE of approximately $300 \mathrm{~s}$ reconstruction of PEDs. Only around six modes produced by HODMDc or THDMDc in Figure 12a can be considered permanent, with low absolute damping $|\delta|<0.01$. Many modes show slight transient behaviour, with $|\delta|>0.01$.

Many amplitudes of the selected fifteen HODMDc and THDMDc modes in Figure 12b are high, with several modes of amplitude between ten and 100. All modes have amplitude greater than 0.1. The modes of lowest amplitude, less than one in this case, may be considered as noise. Modes show negative correlation of frequency and amplitude. A greater number of HODMDc than THDMDc modes are of amplitude low enough to be regarded as noise.

Reconstruction and prediction results using the five modes of highest energy index are shown in Figures 13a, and 13b for the German RWE and French PEDs, respectively. Early reconstruction results are especially noisy for the German PED. Neither technique predicts the German peak after 250 s. The THDMDc prediction appears to follow the peaks of the German PED in the final $100 \mathrm{~s}$. The RRMSE of prediction of all eight PEDs is 1.119 using the selected five HODMDc modes and is 1.073 using the selected five THDMDc modes.

The prediction for France fails as neither HODMDc nor THDMDc modes 


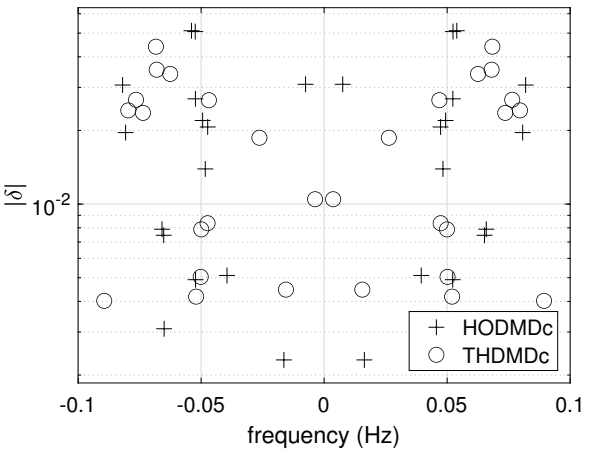

(a)

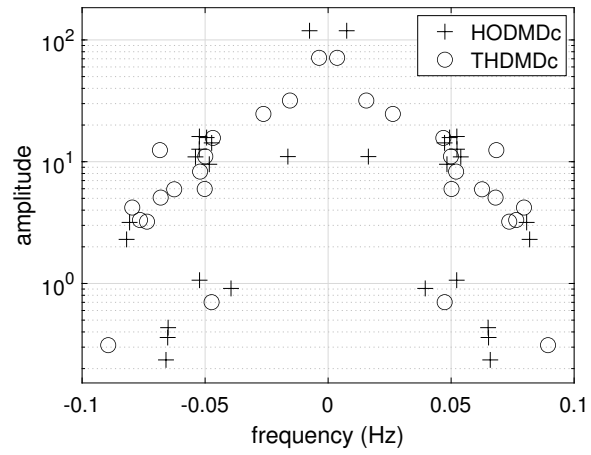

(b)

Figure 12: (a) Absolute damping and (b) amplitudes of 15 HODMDc and THDMDc modes of European PEDs, selected for minimum RRMSE of reconstruction.

capture the sharp decline in French PED during the final $70 \mathrm{~s}$ of the snapshot window. The influence of final snapshots is reduced by the delay-embedding used in higher-order techniques HODMDc and THDMDc. Reconstruction of the French PED appears more accurate for THDMDc than HODMDc. Reconstruction RRMSE for the eight European PEDs is 0.478 for the five plotted THDMDc modes, compared to 0.524 for the five plotted HODMDc modes.

The four dominant spatial elements of the first normalized modes correspond to RWE TSO (Germany), France, Italy and Spain via HODMDc and THDMDc in Figures 14a and 14b, respectively. Elements of mode one for Germany and Italy oscillate against the dominant elements for France and Spain in both HODMDc and THDMDc results. The magnitude of these dominant mode elements is similar in both cases, with some difference particularly between the arguments of elements corresponding to Germany and Italy. The PED for France is most dominant in both results for mode one. 


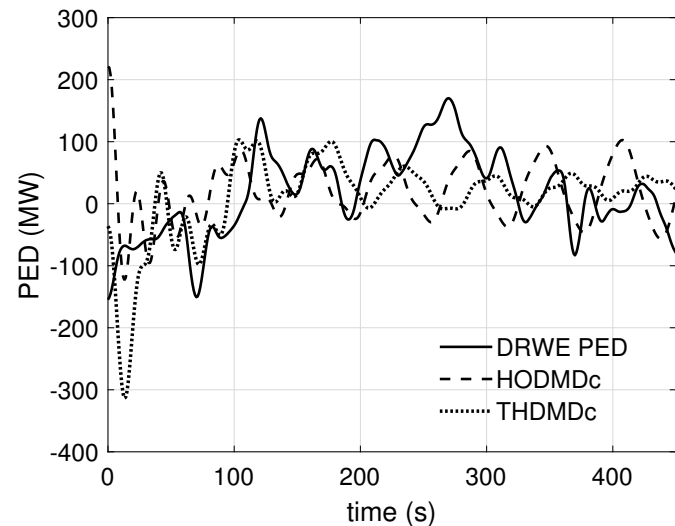

(a)

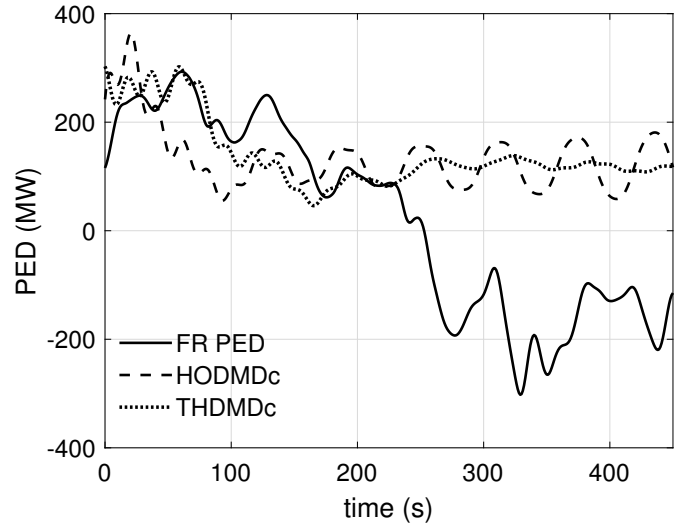

(b)

Figure 13: Reconstruction of $300 \mathrm{~s}$ from 21:50:20 h and prediction for $150 \mathrm{~s}$ of the PEDs for (a) German RWE and (b) France using five HODMDc and THDMDc modes.

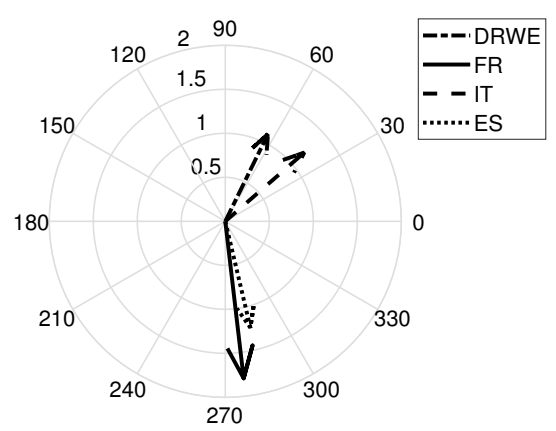

(a)

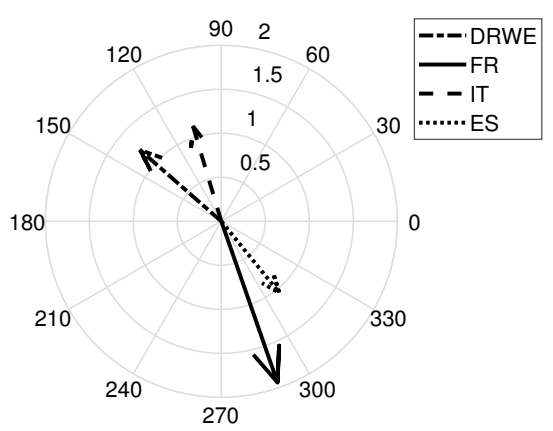

(b)

Figure 14: Compass plots of four dominant components of (a) the first HODMDc mode and (b) the first THDMDc mode of European PEDs. 


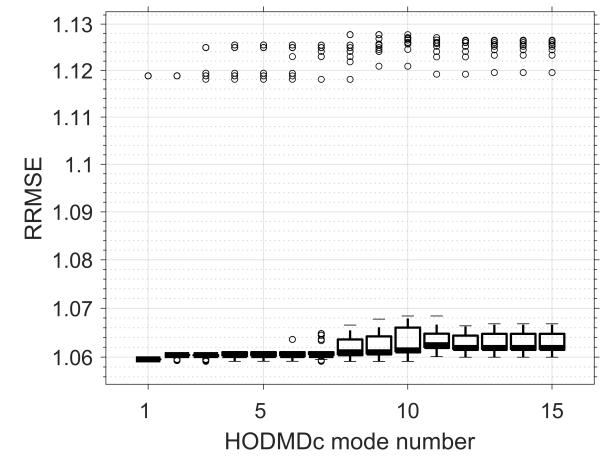

(a)

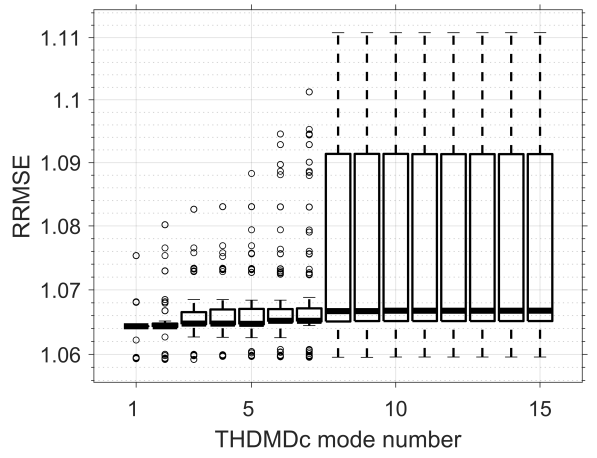

(b)

Figure 15: RRMSE of prediction for (a) 15 HODMDc modes and (b) 15 THDMDc modes of European PEDs. Median, quartiles and outliers (circles) are shown for 300 mode clustering iterations

Although prediction results for France fail to capture transient dynamics necessary for prediction, the dominance of France in mode one in Figures 14a and 14b agrees with the large PEDs for France shown in [33].

Relative RMS error of prediction results are shown for all 300 mode clustering iterations for HODMDc in Figure 15a and for THDMDc in Figure 15b. Median RRMSE results are similar, although RRMSE of prediction is generally slightly higher for THDMDc than for HODMDc, particularly using eight or more modes. 


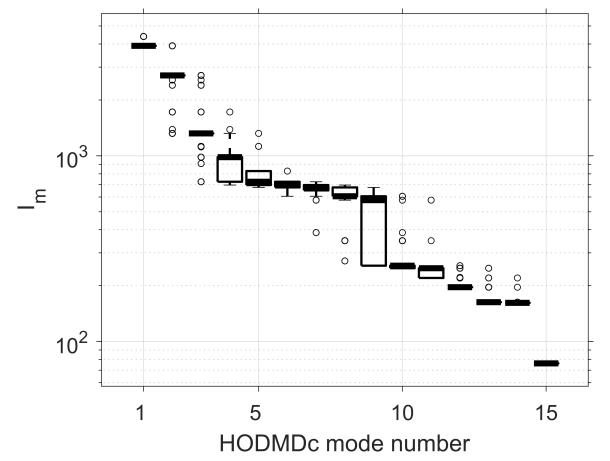

(a)

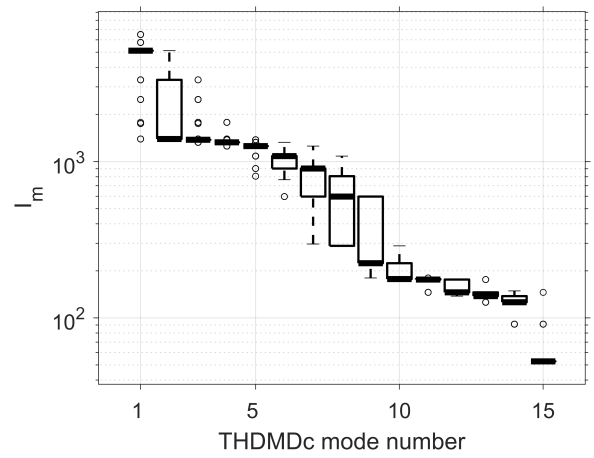

(b)

Figure 16: Energy index, $I_{m}$, for (a) 15 HODMDc modes and (b) 15 THDMDc modes of European PEDs. Median, quartiles and outliers (circles) are shown for 300 mode clustering iterations.

Energy indices for all clustering results of fifteen HODMDc modes and fifteen THDMDc modes are shown in Figures 16a and 16b, respectively. The figures reveal that the median modal energy indices are higher for the THDMDc modes than the HODMDc modes, up to mode eight. Median energy index for HODMDc mode one is almost 4000, whilst the energy index of THDMDc mode one is over 5100. Median energy indices of THDMDc modes two to five are almost constant, above 1000. The first five HODMDc median modal energy indices decline gradually, on the logarithmic scale, to 725 for mode five. Modal energy indices are low for modes ten and above for both techniques, falling below 100 for mode fifteen.

The European UCTE Final Report [33] particularly noted that strong winds and high wind power output in Germany were of great concern at the time of the blackout. German wind power is commonly exported to southern parts of Europe but at the time of the outage, transmission lines were highly 
loaded [33]. This high wind power generation was a significant factor in the cascading outage [33]. The PED for France has a greater range of values during the prediction time than the RWE German PED spans. Both first modes using HODMDc and THDMDc find the contribution of the German PED to be one of the four dominant elements. The oscillation of Germany against France and Spain reflects the common North-South European mode, known often to be caused by export of German wind power. German and Spanish modal elements are almost exactly out of phase, with an angular difference of almost $180^{\circ}$ in THDMDc mode one.

Using a relatively low parameter $d$ for delay-embedding of the snapshots, both HODMDc and THDMDc can approximate some oscillations of noisy snapshots. Using a relatively high SVD threshold $\epsilon_{S V D}$, with a lower projection SVD threshold $\epsilon_{\mathbb{P}}$, THDMDc modes especially can be selected to form an approximate reconstruction and prediction. Prediction results can fail, however, in cases of strong transient behaviour. This can particularly concern cases of transition to a new steady state, towards the end of a given sampling window.

\section{Conclusion}

Higher-order Koopman techniques, HODMDc and THDMDc, have been used for modal analysis and short-term prediction of power systems. Datadriven modal analysis in this work uses noisy measurements recorded before, or during, historical blackout events. Eigenvalues, produced by multiple applications of HODMDc and THDMDc to randomly shifted snapshot windows, are clustered for selection. Corresponding modes are fitted to a Gaussian 
model, to reduce dependence on the sampling window and to identify modes suitable for prediction.

Smooth or multiple transitions in RRMSE of SVD reconstruction of the snapshots suggest that noise can have a range of values. Parameters are set for consistently low RRMSE of reconstruction, for a range of $d$ at a given SVD threshold. Inclusion of very small singular values can overfit noisy snapshots and can introduce artefacts of the sampling window. Very small singular values can also cause severe ill-conditioning. The SVD threshold is therefore selected to retain a physically meaningful decomposition with sufficient spectral complexity and denoising. This approach can provide a short-term prediction close to original values.

Using a maximum of 20 HODMDc modes, analysis for a range of SVD thresholds and settings of $d$ reveals that the RRMSE of prediction can vary by orders of magnitude. The RRMSE of HODMDc prediction can be several times higher with lower or higher SVD thresholds than the moderate value, selected for low RRMSE of reconstruction and locally low sensitivity to $d$. With calibrated parameter settings, prediction can still fail in cases of extreme transient behaviour, which can involve external forcing.

Absolute damping values and amplitudes of some modes can be greater with THDMDc than HODMDc, due to the total-least-squares reduction of noise bias error. Prediction results of all modal clusters can also span a lower range of RRMSE values using THDMDc, compared to HODMDc. Depending on the levels of the noise, the two techniques can produce very similar modal results. Prediction can be superior using THDMDc, which requires future study and systematic analysis of a wider range of disturbance events. 
The obtained results demonstrate that HODMDc and THDMDc can identify the frequencies and damping of oscillations within a short sampling window of several minutes. Without governing equations specific to generators, data-driven modal elements can identify contributions of renewable sources of energy prior to, or during, power system disturbances.

For power system stability analysis, poorly damped modes of the true system dynamics must be recognized. Future work will be focused on further reduction of noise using alternative variants of these higher-order Koopman techniques, especially of THDMD for mitigation of bias error due to noise.

\section{Acknowledgment}

The authors would like to acknowledge the assistance given by Research IT and the use of the Computational Shared Facility at The University of Manchester. C. Jones also wishes to acknowledge assistance from Ryan Maguire of The MathWorks Technical Support for general guidance in the use of fitgmdist.m and fcm.m and to thank Adam Danz for font sizing of the compass plots.

The authors are grateful to the anonymous referees for useful remarks that allowed them to improve the quality of this paper.

\section{Appendix A.}




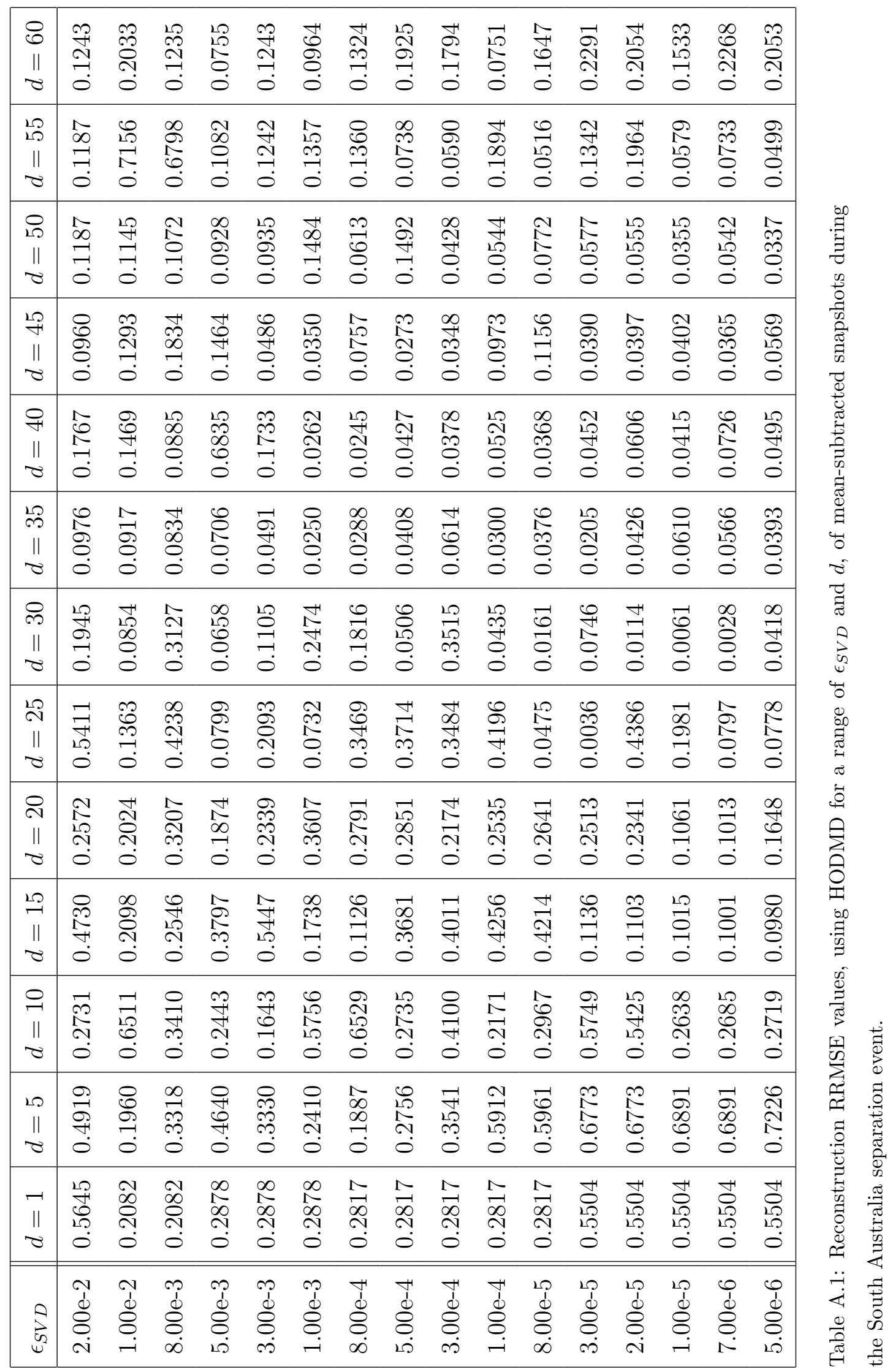




\begin{tabular}{|c|c|c|c|c|c|c|c|c|c|c|c|c|c|c|c|c|}
\hline $\begin{array}{l}8 \\
\| \\
0\end{array}$ & 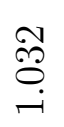 & 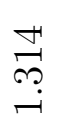 & 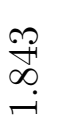 & 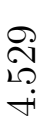 & $\begin{array}{l}\infty \\
\infty \\
\infty \\
\dot{+}\end{array}$ & $\begin{array}{l}\stackrel{\leftrightarrow}{\ominus} \\
\stackrel{\sim}{\sim} \\
\tilde{N}\end{array}$ & $\underset{\sim}{\stackrel{7}{\rightleftarrows}}$ & 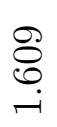 & $\begin{array}{l}\text { vo } \\
\text { ஸे } \\
\text { ণ }\end{array}$ & $\begin{array}{l}\stackrel{P}{N} \\
\stackrel{\leftrightarrow}{\sim} \\
\stackrel{\sim}{N}\end{array}$ & $\begin{array}{l}\text { i⿱ } \\
\text { 군 }\end{array}$ & $\begin{array}{l}\stackrel{N}{+} \\
\stackrel{\sim}{\sim} \\
\text { N }\end{array}$ & & 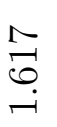 & $\underset{\stackrel{\sim}{\sim}}{\stackrel{+}{*}}$ & $\begin{array}{l}10 \\
0 \\
10 \\
10\end{array}$ \\
\hline 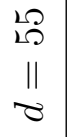 & $\begin{array}{l}\text { ヘิ } \\
\text { ర్} \\
\text {. }\end{array}$ & 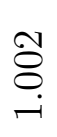 & 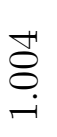 & $\begin{array}{l}\underset{+}{N} \\
\text { r }\end{array}$ & $\begin{array}{l}\stackrel{+2}{7} \\
\stackrel{1}{\sim}\end{array}$ & 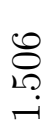 & $\begin{array}{l}\text { बे } \\
\text { ले } \\
\text { † }\end{array}$ & 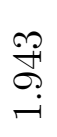 & $\underset{\stackrel{+}{\infty}}{\stackrel{+}{\infty}}$ & ชి & $\begin{array}{l}\underset{\infty}{+} \\
\stackrel{1}{\sim}\end{array}$ & 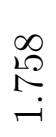 & 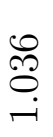 & 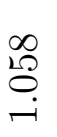 & 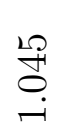 & 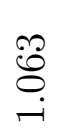 \\
\hline $\begin{array}{l}80 \\
\| \\
\| \\
0\end{array}$ & 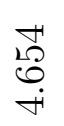 & 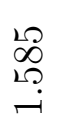 & 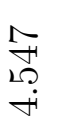 & $\underset{\sim}{\stackrel{\sim}{\sim}}$ & $\underset{\infty}{\stackrel{\rho}{\not}}$ & 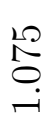 & & 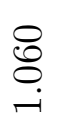 & 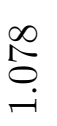 & 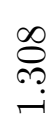 & $\begin{array}{l}\stackrel{\odot}{\infty} \\
\stackrel{\infty}{\sim} \\
\sim\end{array}$ & \begin{tabular}{l}
$\infty$ \\
$\substack{0 \\
\hdashline}$
\end{tabular} & 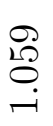 & 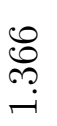 & نُ-ْ & $\underset{\varnothing}{\varnothing}$ \\
\hline $\begin{array}{l}\stackrel{20}{\rightarrow} \\
\underset{\sigma}{\|}\end{array}$ & $\begin{array}{l}12 \\
12 \\
\infty \\
0 \\
0 \\
-1\end{array}$ & 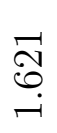 & 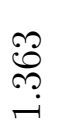 & $\begin{array}{l}\infty \\
\stackrel{1}{2} \\
\stackrel{\sim}{\sim}\end{array}$ & \begin{tabular}{l}
0 \\
$\infty$ \\
$\infty$ \\
\multirow{1}{*}{}
\end{tabular} & $\begin{array}{l}\infty \\
\stackrel{\infty}{-1} \\
-1\end{array}$ & 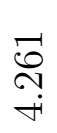 & $\begin{array}{l}\infty \\
\infty \\
0 \\
0\end{array}$ & $\begin{array}{l}\frac{1}{2} \\
0 \\
0\end{array}$ & $\begin{array}{l}1 \\
0 \\
0 \\
0\end{array}$ & $\begin{array}{l}m \\
\dot{0} \\
\dot{0}\end{array}$ & $\begin{array}{l}\infty \\
\infty \\
\infty \\
\infty\end{array}$ & $\begin{array}{l}\stackrel{0}{10} \\
\stackrel{N}{+} \\
\dot{+}\end{array}$ & \begin{tabular}{l}
$\infty$ \\
\multirow{N}{*}{} \\
$\infty$ \\
0
\end{tabular} & $\begin{array}{l}m \\
\stackrel{2}{\sim} \\
\tilde{\sim}\end{array}$ & $\begin{array}{l}\overrightarrow{0} \\
\stackrel{+}{1} \\
\dot{\infty}\end{array}$ \\
\hline $\begin{array}{l}\stackrel{P}{+} \\
\| \\
\sim\end{array}$ & 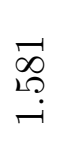 & $\begin{array}{l}\infty \\
\stackrel{\infty}{\sim} \\
\stackrel{\sim}{-}\end{array}$ & 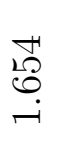 & $\begin{array}{l}0 \\
\stackrel{0}{\sigma} \\
\ddot{-}\end{array}$ & $\begin{array}{l}\infty \\
\stackrel{\infty}{0} \\
\stackrel{N}{v}\end{array}$ & 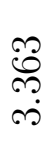 & $\begin{array}{l}\stackrel{o}{N} \\
\text { N } \\
\sim\end{array}$ & 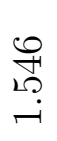 & $\underset{\overbrace{}}{\stackrel{\leftrightarrow}{\leftarrow}}$ & $\begin{array}{l}\underset{N}{N} \\
0\end{array}$ & $\begin{array}{l}8 \\
0 \\
0 \\
0\end{array}$ & $\begin{array}{l}10 \\
\stackrel{1}{\infty} \\
\infty \\
\infty\end{array}$ & $\begin{array}{l}\infty \\
\stackrel{\infty}{\sigma} \\
\dot{\nabla}\end{array}$ & $\begin{array}{l}\stackrel{0}{\infty} \\
\stackrel{\infty}{+!} \\
\stackrel{1}{n}\end{array}$ & $\begin{array}{l}\infty \\
\infty \\
\infty \\
\Lambda\end{array}$ & 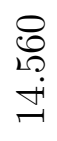 \\
\hline $\begin{array}{l}20 \\
\text { o } \\
\| \\
\sim\end{array}$ & $\begin{array}{l}\stackrel{H}{\sim} \\
\infty \\
\stackrel{\sim}{d}\end{array}$ & $\begin{array}{l}1 \\
0 \\
10 \\
\infty \\
\infty \\
0\end{array}$ & 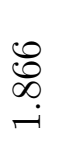 & $\underset{\infty}{\stackrel{20}{\rightleftarrows}}$ & $\begin{array}{l}\text { ㄱ. } \\
\text { in } \\
\stackrel{N}{N}\end{array}$ & $\begin{array}{l}\stackrel{\infty}{+} \\
\stackrel{\infty}{\infty} \\
\infty\end{array}$ & 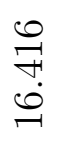 & $\begin{array}{l}10 \\
\infty \\
0 \\
1 \\
10\end{array}$ & $\frac{N}{\tilde{N}}$ & 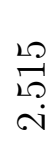 & 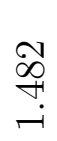 & 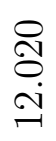 & 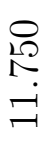 & $\begin{array}{l}10 \\
10 \\
\infty \\
10 \\
10\end{array}$ & $\begin{array}{l}\infty \\
\stackrel{+}{+} \\
\stackrel{0}{\circ}\end{array}$ & $\begin{array}{l}\text { î } \\
\text { 룽 }\end{array}$ \\
\hline 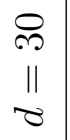 & $\begin{array}{l}\infty \\
\stackrel{\sim}{\sim} \\
\stackrel{\sim}{\sim}\end{array}$ & $\begin{array}{l}\text { gे } \\
\text { ळे }\end{array}$ & 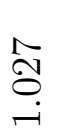 & $\begin{array}{l}\stackrel{\gamma}{\delta} \\
\infty \\
\dot{0}\end{array}$ & & 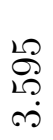 & 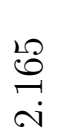 & $\begin{array}{l}0 \\
\stackrel{0}{\infty} \\
\infty \\
\sim\end{array}$ & $\begin{array}{l}\text { 足 } \\
\stackrel{2}{\sim}\end{array}$ & $\begin{array}{l}1 \\
0 \\
10 \\
ن \\
ن\end{array}$ & $\begin{array}{l}\stackrel{N}{N} \\
\stackrel{N}{*} \\
\text { ஸे }\end{array}$ & 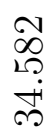 & 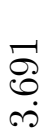 & $\begin{array}{l}12 \\
20 \\
\infty \\
\stackrel{-1}{-1}\end{array}$ & $\begin{array}{l}\stackrel{+}{N} \\
\stackrel{\sim}{\sim}\end{array}$ & 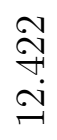 \\
\hline $\begin{array}{l}\stackrel{2}{\sim} \\
\| \\
\stackrel{\sim}{\|}\end{array}$ & 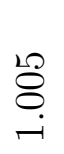 & 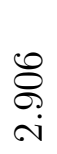 & $\begin{array}{l}1 \\
\infty \\
0 \\
0\end{array}$ & $\begin{array}{l}\infty \\
\infty \\
\infty \\
0 \\
0\end{array}$ & $\stackrel{\stackrel{L}{O}}{0}$ & 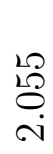 & $\underset{\leftarrow}{\stackrel{\sim}{\rightleftarrows}}$ & \begin{tabular}{l}
$\infty$ \\
10 \\
0 \\
\hdashline \\
-1
\end{tabular} & $\underset{\sim}{\stackrel{P}{+}}$ & 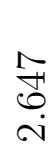 & $\begin{array}{l}\stackrel{L}{O} \\
\text { \% } \\
\stackrel{1}{-1}\end{array}$ & 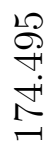 & $\begin{array}{l}\text { N } \\
\text { ำ } \\
\text { Ñ} \\
\text { ָे }\end{array}$ & $\begin{array}{l}\vec{\infty} \\
\infty \\
\dot{\infty} \\
\infty\end{array}$ & 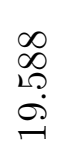 & $\begin{array}{l}\stackrel{8}{2} \\
\stackrel{1}{7} \\
\stackrel{1}{\sim}\end{array}$ \\
\hline $\begin{array}{c}\stackrel{\sim}{\Omega} \\
\| \\
\sim\end{array}$ & $\begin{array}{l}\overrightarrow{D D} \\
\vec{A}\end{array}$ & $\underset{\sigma}{\sigma}$ & $\underset{\square}{\stackrel{+}{\sigma}}$ & $\begin{array}{l}\text { Sू } \\
\text { S }\end{array}$ & $\underset{\stackrel{H}{\sim}}{\stackrel{\sim}{\sim}}$ & $\stackrel{\mathscr{H}}{\stackrel{\rho}{0}}$ & 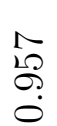 & 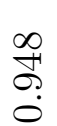 & $\begin{array}{l}\bar{b} \\
\text { ஸִ } \\
+\end{array}$ & 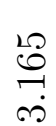 & 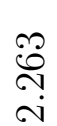 & $\begin{array}{l}\stackrel{0}{2} \\
\stackrel{2}{n} \\
\end{array}$ & $\begin{array}{l}\infty \\
\stackrel{2}{\sim} \\
\stackrel{\sim}{\sim}\end{array}$ & 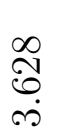 & $\begin{array}{l}\overrightarrow{1} \\
\infty \\
0 \\
0\end{array}$ & $\begin{array}{l}\stackrel{20}{\sim} \\
\stackrel{\sim}{\infty} \\
\infty\end{array}$ \\
\hline$\underset{⿱ ⺌}{\stackrel{L O}{\pi}}$ & 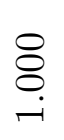 & $\frac{8}{\mathscr{2}}$ & $\begin{array}{l}\stackrel{H}{2} \\
\stackrel{2}{0} \\
0\end{array}$ & 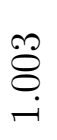 & 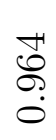 & 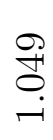 & 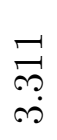 & $\begin{array}{l}\infty \\
\infty \\
\infty \\
0\end{array}$ & 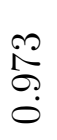 & $\begin{array}{l}\infty \\
\stackrel{2}{0} \\
0 \\
0\end{array}$ & $\begin{array}{l}\stackrel{10}{+} \\
\stackrel{0}{0}\end{array}$ & $\begin{array}{l}\overline{0} \\
0 \\
0\end{array}$ & $\begin{array}{l}10 \\
2 \\
0 \\
0\end{array}$ & $\begin{array}{l}\stackrel{10}{\stackrel{5}{0}} \\
\stackrel{0}{0}\end{array}$ & $\begin{array}{l}\Gamma \\
\text { Oे } \\
0\end{array}$ & $\begin{array}{l}10 \\
0 \\
0 \\
0 \\
0\end{array}$ \\
\hline $\begin{array}{l}\stackrel{\rho}{-} \\
\| \\
\stackrel{\sigma}{\sigma}\end{array}$ & 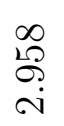 & $\underset{8}{8}$ & $\underset{\sim}{\stackrel{8}{\varnothing}}$ & 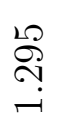 & $\begin{array}{l}\underset{\sim}{H} \\
\text { v }\end{array}$ & $\stackrel{\overbrace{}}{\stackrel{\circ}{\circ}}$ & 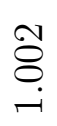 & $\begin{array}{l}\stackrel{H}{N} \\
\stackrel{N}{N}\end{array}$ & 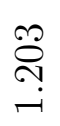 & 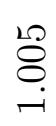 & 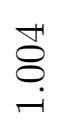 & 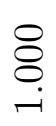 & $\underset{ْ}{\varnothing}$ & $\underset{\mathscr{\theta}}{\mathscr{8}}$ & $\underset{ْ}{\mathscr{Q}}$ & $\underset{\varnothing}{\mathscr{\varnothing}}$ \\
\hline $\begin{array}{c}10 \\
\| \\
\nabla\end{array}$ & $\begin{array}{l}\stackrel{+}{\mathbb{2}} \\
\stackrel{0}{0}\end{array}$ & $\begin{array}{l}\stackrel{\sim}{\ominus} \\
\stackrel{\sim}{\sim}\end{array}$ & 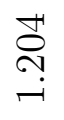 & $\begin{array}{c}\text { N } \\
\text { O }\end{array}$ & ๙ิ & ְ. & $\begin{array}{l}\stackrel{\circ}{+} \\
\underset{\sim}{\sim}\end{array}$ & $\begin{array}{l}\stackrel{N}{\sim} \\
\stackrel{\leftrightarrow}{\sim} \\
\stackrel{1}{*}\end{array}$ & $\underset{\varnothing}{\stackrel{+}{\varnothing}}$ & $\underset{\overbrace{}}{\varnothing}$ & $\begin{array}{l}0 \\
\infty \\
0 \\
0\end{array}$ & 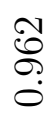 & 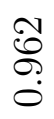 & $\begin{array}{l}\stackrel{0}{0} \\
\stackrel{0}{0}\end{array}$ & $\begin{array}{l}? \\
\stackrel{0}{0} \\
0\end{array}$ & $\begin{array}{l}\stackrel{N}{N} \\
\stackrel{?}{0}\end{array}$ \\
\hline$\underset{\sim}{\pi}$ & & $\begin{array}{l}\infty \\
\stackrel{N}{L} \\
\end{array}$ & $\frac{\infty}{N}$ & $\underset{\stackrel{+}{\sim}}{\stackrel{\infty}{+}}$ & $\underset{\sim}{\stackrel{\infty}{\underset{H}{+}}}$ & $\underset{\sim}{\stackrel{\infty}{+}}$ & $\underset{\sim}{\sim}$ & $\underset{\sim}{\stackrel{F}{\sim}}$ & $\underset{\sim}{\sim}$ & $\underset{\sim}{\sim}$ & $\underset{\sim}{\stackrel{\sim}{\sim}}$ & 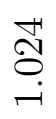 & $\underset{\sim}{\stackrel{+}{\sim}}$ & 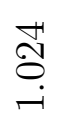 & 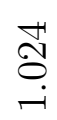 & 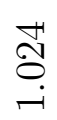 \\
\hline $\begin{array}{l}\vec{A} \\
\underbrace{2}\end{array}$ & 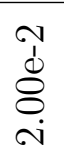 & 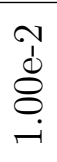 & $\begin{array}{c}\infty \\
\dot{d} \\
\stackrel{8}{0} \\
\infty\end{array}$ & $\begin{array}{c}\infty \\
\dot{d} \\
8 \\
\delta \\
\dot{0}\end{array}$ & 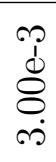 & 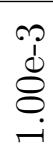 & $\underset{\infty}{\stackrel{+}{d}}$ & $\begin{array}{c}\overrightarrow{1} \\
\stackrel{d}{8} \\
\stackrel{0}{\delta} \\
\dot{0}\end{array}$ & $\underset{\infty}{\stackrel{+}{d}}$ & $\begin{array}{l}\vec{j} \\
\stackrel{d}{d} \\
\stackrel{0}{0}\end{array}$ & 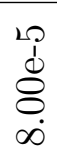 & $\begin{array}{c}20 \\
\dot{d} \\
8 \\
8 \\
\infty\end{array}$ & 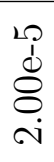 & $\begin{array}{l}10 \\
d \\
8 \\
8 \\
\end{array}$ & 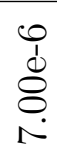 & $\begin{array}{c}0 \\
1 \\
\delta \\
\delta \\
0\end{array}$ \\
\hline
\end{tabular}




\begin{tabular}{|c|c|c|c|c|c|c|c|c|c|c|c|c|c|c|c|c|}
\hline 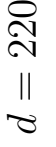 & $\begin{array}{l}10 \\
\stackrel{10}{10} \\
\stackrel{0}{0}\end{array}$ & $\begin{array}{l}10 \\
0 \\
0 \\
0 \\
0\end{array}$ & $\underset{\dot{D}}{\stackrel{N}{+}}$ & $\frac{2}{\stackrel{2}{0}}$ & $\begin{array}{l}0 \\
\stackrel{0}{0} \\
0\end{array}$ & 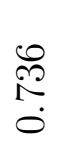 & i⿱ & $\begin{array}{l}\stackrel{0}{10} \\
\stackrel{1}{0}\end{array}$ & $\stackrel{\stackrel{L}{+}}{\stackrel{1}{+}}$ & $\begin{array}{l}\stackrel{9}{+} \\
\stackrel{0}{0}\end{array}$ & 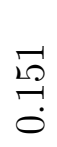 & $\stackrel{10}{\stackrel{10}{0}}$ & $\begin{array}{l}\infty \\
\text { 年 } \\
\text { iी } \\
0\end{array}$ & $\begin{array}{l}10 \\
\stackrel{10}{0} \\
\ddot{0}\end{array}$ & 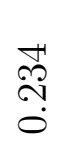 & $\underset{\dot{0}}{\stackrel{N}{D}}$ \\
\hline $\begin{array}{l}\stackrel{0}{\vec{J}} \\
\| \\
\|\end{array}$ & $\begin{array}{l}0 \\
\stackrel{\Omega}{0} \\
0\end{array}$ & $\begin{array}{l}\stackrel{0}{1} \\
\infty \\
\dot{0}\end{array}$ & $\begin{array}{l}0 \\
+ \\
\dot{H}\end{array}$ & \begin{tabular}{l}
$\infty$ \\
$\infty$ \\
\multirow{0}{0}{} \\
0
\end{tabular} & 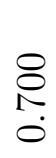 & $\begin{array}{l}\stackrel{2}{N} \\
\stackrel{0}{0}\end{array}$ & $\begin{array}{l}\vec{\delta} \\
\infty \\
\dot{0}\end{array}$ & $\begin{array}{l}\stackrel{2}{1} \\
\stackrel{0}{0}\end{array}$ & $\begin{array}{l}\stackrel{20}{20} \\
\stackrel{0}{0}\end{array}$ & $\underset{⿱ N}{\stackrel{N}{0}}$ & $\begin{array}{l}\text { N } \\
\stackrel{0}{\circ}\end{array}$ & $\begin{array}{l}\stackrel{0}{0} \\
\stackrel{0}{0}\end{array}$ & $\begin{array}{l}\stackrel{8}{0} \\
\varrho \\
ٌ\end{array}$ & $\begin{array}{l}\stackrel{\Omega}{1} \\
\stackrel{0}{0} \\
\stackrel{0}{0}\end{array}$ & $\begin{array}{l}\mathfrak{N} \\
\stackrel{0}{0} \\
0\end{array}$ & $\begin{array}{l}0 \\
\text { mo } \\
0\end{array}$ \\
\hline 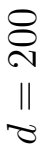 & $\begin{array}{l}\vec{\infty} \\
\infty \\
\dot{0}\end{array}$ & $\begin{array}{l}\stackrel{10}{10} \\
\stackrel{0}{0} \\
0\end{array}$ & $\begin{array}{l}\overrightarrow{0} \\
0 \\
0\end{array}$ & $\stackrel{0}{\underline{1}}$ & $\begin{array}{l}\stackrel{2}{N} \\
\stackrel{i}{0}\end{array}$ & $\begin{array}{l}\text { ⿵ె } \\
\text { రై } \\
0\end{array}$ & $\begin{array}{l}\text { ஸे } \\
\text { రై } \\
\dot{0}\end{array}$ & 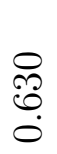 & $\begin{array}{l}\mathscr{\leftrightarrow} \\
\stackrel{0}{0} \\
\ddot{0}\end{array}$ & $\begin{array}{l}\infty \\
\stackrel{N}{0} \\
0 \\
0\end{array}$ & $\begin{array}{l}0 \\
\text { ֶै } \\
\stackrel{0}{0}\end{array}$ & $\begin{array}{l}\stackrel{\vec{J}}{\sigma} \\
\stackrel{0}{0}\end{array}$ & $\begin{array}{l}\overparen{6} \\
\stackrel{0}{0}\end{array}$ & $\begin{array}{l}\overparen{0} \\
\stackrel{0}{0} \\
0\end{array}$ & $\begin{array}{l}8 \\
\stackrel{8}{0} \\
0\end{array}$ & $\begin{array}{l}\mathscr{L} \\
\mathscr{O} \\
\stackrel{0}{0}\end{array}$ \\
\hline $\begin{array}{c}\underset{\Omega}{\sigma} \\
\| \\
\sim\end{array}$ & $\begin{array}{l}\vec{\Omega} \\
\stackrel{0}{0}\end{array}$ & $\begin{array}{l}\infty \\
\infty \\
⿱ \\
0\end{array}$ & $\begin{array}{l}0 \\
\stackrel{1}{2} \\
0\end{array}$ & $\begin{array}{l}R \\
\stackrel{R}{0}\end{array}$ & $\begin{array}{l}\stackrel{0}{\infty} \\
1 \\
0\end{array}$ & $\begin{array}{l}1 \\
\infty \\
1 \\
0\end{array}$ & $\begin{array}{l}\stackrel{10}{O} \\
0 \\
0\end{array}$ & $\begin{array}{l}\mathscr{D} \\
\stackrel{0}{0}\end{array}$ & $\begin{array}{l}\vec{N} \\
\stackrel{N}{0}\end{array}$ & $\begin{array}{l}1 \\
0 \\
0 \\
0\end{array}$ & $\begin{array}{l}\stackrel{+}{D} \\
0\end{array}$ & $\begin{array}{l}\overrightarrow{0} \\
\stackrel{+}{0}\end{array}$ & $\begin{array}{l}100 \\
\stackrel{10}{0} \\
0\end{array}$ & $\begin{array}{l}\infty \\
\stackrel{1}{0} \\
\stackrel{0}{0} \\
0\end{array}$ & 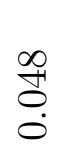 & $\begin{array}{l}\infty \\
0 \\
0\end{array}$ \\
\hline $\begin{array}{l}\infty \\
\underset{\sim}{\infty} \\
\| \\
\nabla\end{array}$ & $\begin{array}{l}\overrightarrow{0} \\
0 \\
0\end{array}$ & $\begin{array}{l}\infty \\
\infty \\
\stackrel{0}{0} \\
0\end{array}$ & $\underset{0}{\stackrel{\infty}{0}}$ & $\begin{array}{l}\stackrel{2}{N} \\
\stackrel{0}{0}\end{array}$ & $\stackrel{N}{\stackrel{n}{0}}$ & $\underset{0}{\vec{H}}$ & $\stackrel{10}{\Gamma}$ & $\stackrel{0}{\stackrel{0}{二}}$ & $\begin{array}{l}\text { Lo } \\
\stackrel{0}{0}\end{array}$ & $\begin{array}{l}\mathscr{N} \\
0 \\
0 \\
0\end{array}$ & $\begin{array}{l}\stackrel{0}{10} \\
\stackrel{1}{0} \\
0\end{array}$ & 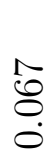 & $\begin{array}{l}0 \\
\varrho \\
ٌ \\
0\end{array}$ & $\begin{array}{l}\stackrel{\leftrightarrow}{0} \\
\stackrel{0}{0} \\
0\end{array}$ & $\begin{array}{l}\stackrel{\Im}{0} \\
\stackrel{0}{0} \\
0\end{array}$ & $\underset{0}{\stackrel{N}{0}}$ \\
\hline $\begin{array}{l}\stackrel{P}{\sim} \\
\| \\
\sim \\
\sim\end{array}$ & $\begin{array}{l}\stackrel{\sim}{\sigma} \\
\stackrel{\sim}{\circ} \\
\stackrel{0}{0}\end{array}$ & $\begin{array}{l}\infty \\
\stackrel{\infty}{\sim} \\
\stackrel{0}{0}\end{array}$ & $\begin{array}{l}0 \\
\stackrel{\infty}{1} \\
0\end{array}$ & $\begin{array}{l}\infty \\
\stackrel{0}{\infty} \\
0\end{array}$ & $\begin{array}{l}+ \\
\stackrel{8}{\circ} \\
0\end{array}$ & $\begin{array}{l}0 \\
\stackrel{R}{1} \\
0\end{array}$ & $\begin{array}{l}\text { 금 } \\
\stackrel{\sim}{0}\end{array}$ & 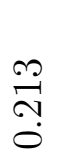 & $\begin{array}{l}\stackrel{\leftrightarrow}{1} \\
0 \\
0\end{array}$ & $\begin{array}{l}\stackrel{10}{0} \\
\stackrel{0}{0} \\
0\end{array}$ & $\begin{array}{l}\stackrel{6}{\circ} \\
\stackrel{\circ}{\circ}\end{array}$ & $\begin{array}{l}\stackrel{1}{\sigma} \\
\stackrel{0}{0}\end{array}$ & $\begin{array}{l}\stackrel{\sim}{\beth} \\
\stackrel{0}{0}\end{array}$ & $\begin{array}{l}\stackrel{\sim}{\rightleftharpoons} \\
\stackrel{0}{\circ}\end{array}$ & $\stackrel{\infty}{\stackrel{\infty}{\not}}$ & $\begin{array}{l}\infty \\
\stackrel{\infty}{0} \\
\stackrel{0}{0}\end{array}$ \\
\hline 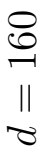 & $\underset{\nabla}{\check{\sigma}}$ & $\underset{\stackrel{H}{H}}{\stackrel{H}{0}}$ & $\stackrel{\stackrel{\mathscr{N}}{\oplus}}{\stackrel{\leftrightarrow}{0}}$ & 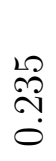 & 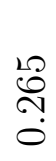 & $\begin{array}{l}\stackrel{N}{N} \\
\stackrel{N}{0}\end{array}$ & $\begin{array}{c}\stackrel{\oplus}{N} \\
\stackrel{\sim}{0}\end{array}$ & $\begin{array}{l}\stackrel{0}{10} \\
\stackrel{0}{0} \\
\stackrel{0}{0}\end{array}$ & $\begin{array}{l}\stackrel{\leftrightarrow}{\sim} \\
\stackrel{\leftrightarrow}{\circ}\end{array}$ & $\begin{array}{l}\stackrel{N}{N} \\
\stackrel{0}{0}\end{array}$ & $\frac{\infty}{\infty}$ & $\frac{120}{\infty}$ & $\begin{array}{l}\mathscr{2} \\
\infty \\
\stackrel{0}{0}\end{array}$ & $\begin{array}{l}\vec{\infty} \\
\stackrel{1}{0}\end{array}$ & $\begin{array}{l}\frac{\infty}{N} \\
\stackrel{!}{0}\end{array}$ & $\begin{array}{l}\stackrel{N}{N} \\
\stackrel{0}{0}\end{array}$ \\
\hline 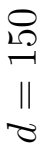 & 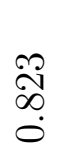 & $\begin{array}{l}\stackrel{2}{N} \\
\stackrel{0}{0}\end{array}$ & $\begin{array}{l}\vec{N} \\
\dot{N}\end{array}$ & $\begin{array}{l}\stackrel{2}{0} \\
\stackrel{0}{0} \\
0\end{array}$ & $\begin{array}{l}\infty \\
\infty \\
\stackrel{0}{0} \\
0\end{array}$ & $\begin{array}{l}8 \\
\text { ? } \\
0 \\
0\end{array}$ & $\begin{array}{l}\vec{H} \\
\dot{0} \\
\dot{0}\end{array}$ & $\begin{array}{l}\stackrel{1}{0} \\
0 \\
0 \\
0\end{array}$ & $\begin{array}{l}\vec{\vartheta} \\
0 \\
0 \\
0\end{array}$ & $\begin{array}{l}10 \\
\infty \\
10 \\
0\end{array}$ & $\begin{array}{l}0 \\
0 \\
0 \\
0\end{array}$ & $\begin{array}{l}1 \\
\text { o } \\
0 \\
0\end{array}$ & $\begin{array}{l}\infty \\
\infty \\
10 \\
0\end{array}$ & $\begin{array}{l}\vec{\infty} \\
10 \\
0 \\
0\end{array}$ & $\begin{array}{l}\text { ov } \\
\infty \\
10 \\
0 \\
0\end{array}$ & $\begin{array}{l}\text { ov } \\
\text { L? } \\
0\end{array}$ \\
\hline $\begin{array}{l}\stackrel{\rho}{+} \\
\| \\
\stackrel{\gamma}{\|}\end{array}$ & $\begin{array}{l}\stackrel{H}{N} \\
\infty \\
\infty \\
0\end{array}$ & $\begin{array}{l}20 \\
\infty \\
0 \\
0\end{array}$ & $\begin{array}{l}\infty \\
\infty \\
\infty \\
\dot{0}\end{array}$ & $\begin{array}{l}0 \\
0 \\
\infty \\
0\end{array}$ & $\begin{array}{l}\stackrel{+}{0} \\
\infty \\
0\end{array}$ & $\begin{array}{l}\stackrel{N}{二} \\
\dot{\infty} \\
\dot{0}\end{array}$ & $\begin{array}{l}\text { No } \\
\infty \\
0 \\
0\end{array}$ & $\begin{array}{l}\infty \\
\infty \\
\infty \\
\dot{0}\end{array}$ & $\begin{array}{l}\stackrel{1}{\infty} \\
\infty \\
\dot{0}\end{array}$ & $\begin{array}{l}\infty 0 \\
\infty \\
0 \\
0\end{array}$ & $\begin{array}{l}\stackrel{10}{-} \\
\stackrel{\infty}{0} \\
\dot{0}\end{array}$ & $\begin{array}{l}0 \\
\stackrel{1}{\infty} \\
\dot{0}\end{array}$ & $\begin{array}{l}\sim \\
\infty \\
\infty \\
0\end{array}$ & $\begin{array}{l}\sim \\
\stackrel{1}{\infty} \\
\stackrel{0}{0}\end{array}$ & $\begin{array}{l}\text { N } \\
\infty \\
0 \\
0\end{array}$ & $\begin{array}{l}N \\
\infty \\
\infty \\
0\end{array}$ \\
\hline $\begin{array}{r}\overrightarrow{1} \\
\vec{\sigma}\end{array}$ & $\underset{\overbrace{}}{\varnothing}$ & $\underset{\overbrace{}}{\stackrel{8}{\&}}$ & $\underset{-}{\varnothing}$ & $\underset{8}{\varnothing}$ & $\underset{ْ}{\varnothing}$ & $\underset{-}{\varnothing}$ & $\underset{\overbrace{}}{\stackrel{8}{\&}}$ & $\underset{\overbrace{}}{\stackrel{8}{8}}$ & $\underset{\overbrace{}}{\varnothing}$ & $\underset{\overbrace{}}{\varnothing}$ & $\underset{-}{\varnothing}$ & $\underset{\&}{8}$ & 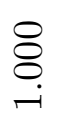 & $\underset{\text { }}{\stackrel{8}{\varnothing}}$ & $\underset{\overbrace{}}{\stackrel{8}{\&}}$ & $\underset{-}{\varnothing}$ \\
\hline 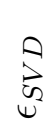 & $\begin{array}{l}\text { d } \\
d \\
0 \\
0\end{array}$ & $\begin{array}{c}\text { d } \\
d \\
ٍ \\
\dot{\forall}\end{array}$ & 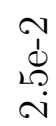 & $\begin{array}{c}v \\
1 \\
0 \\
0 \\
d\end{array}$ & 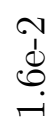 & 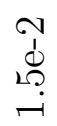 & 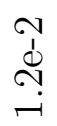 & 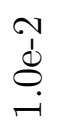 & $\begin{array}{l}m_{0} \\
0 \\
0 \\
0\end{array}$ & $\begin{array}{l}\infty \\
d \\
0 \\
0\end{array}$ & $\begin{array}{c}\infty_{0}^{p} \\
0 \\
0 \\
0\end{array}$ & 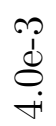 & 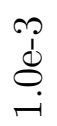 & $\begin{array}{l}\stackrel{+}{d} \\
\stackrel{d}{0}\end{array}$ & 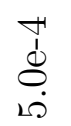 & $\begin{array}{l}\infty \\
1 \\
0 \\
0 \\
-\end{array}$ \\
\hline
\end{tabular}




\begin{tabular}{|c|c|c|c|c|c|c|c|c|c|c|c|c|c|c|c|c|c|c|}
\hline \begin{tabular}{l}
8 \\
$\|$ \\
$\mho$ \\
\hdashline
\end{tabular} & $\begin{array}{l}\stackrel{ }{~} \\
\vec{H} \\
\stackrel{0}{0}\end{array}$ & $\begin{array}{l}\stackrel{H}{S} \\
\text { S. } \\
\stackrel{0}{0}\end{array}$ & $\begin{array}{l}20 \\
20 \\
\infty \\
\stackrel{0}{0} \\
0\end{array}$ & $\begin{array}{l}\stackrel{\infty}{\infty} \\
\underset{\infty}{1} \\
\ddot{0}\end{array}$ & $\begin{array}{l}\vec{b} \\
1 \\
\overrightarrow{0}\end{array}$ & 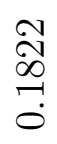 & 疍 & 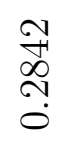 & $\begin{array}{l}\vec{\sigma} \\
\vec{\sigma}\end{array}$ & 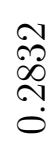 & $\begin{array}{l}\stackrel{R}{N} \\
\stackrel{\sim}{0} \\
\stackrel{0}{0}\end{array}$ & 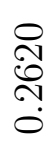 & $\begin{array}{l}\mathscr{S} \\
\infty \\
\stackrel{0}{0}\end{array}$ & $\begin{array}{l}10 \\
\stackrel{10}{8} \\
\stackrel{2}{0}\end{array}$ & $\begin{array}{l}0 \\
0 \\
\stackrel{0}{0} \\
\stackrel{0}{0}\end{array}$ & $\begin{array}{l}\text { 개 } \\
\stackrel{\sim}{0} \\
\stackrel{0}{0}\end{array}$ & $\begin{array}{l}\infty \\
\stackrel{1}{\infty} \\
\sim \\
\stackrel{2}{0} \\
\stackrel{0}{0}\end{array}$ & $\begin{array}{l}\stackrel{g}{+} \\
\stackrel{N}{\sim} \\
\stackrel{0}{0}\end{array}$ \\
\hline $\begin{array}{l}10 \\
11 \\
\sigma \\
\sigma\end{array}$ & $\begin{array}{l}\vec{\infty} \\
\infty \\
\infty \\
0 \\
0\end{array}$ & $\begin{array}{l}\text { iv } \\
\text { i⿱ } \\
\stackrel{0}{0}\end{array}$ & $\begin{array}{l}\hat{N} \\
\stackrel{N}{N} \\
\stackrel{0}{0}\end{array}$ & $\begin{array}{l}\infty \\
\stackrel{8}{0} \\
\sim \\
ٍ \\
0\end{array}$ & 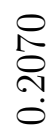 & $\begin{array}{l}\stackrel{\infty}{\underset{N}{N}} \\
\stackrel{\sim}{0}\end{array}$ & 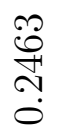 & 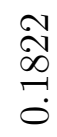 & 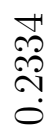 & $\begin{array}{l}\vec{N} \\
\stackrel{\leftrightarrow}{\sim} \\
\stackrel{0}{0}\end{array}$ & 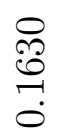 & $\begin{array}{l}m \\
\stackrel{\infty}{\infty} \\
\stackrel{1}{0}\end{array}$ & $\begin{array}{l}\mathscr{0} \\
10 \\
\stackrel{10}{0} \\
0\end{array}$ & $\begin{array}{l}\stackrel{\sim}{*} \\
\stackrel{\sim}{\sim} \\
\stackrel{0}{\circ}\end{array}$ & 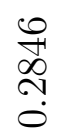 & 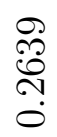 & $\begin{array}{l}\vec{I} \\
\stackrel{2}{20} \\
\stackrel{2}{0}\end{array}$ & 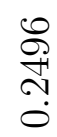 \\
\hline $\begin{array}{l}0 \\
i n \\
\| \\
0 \\
0\end{array}$ & $\begin{array}{l}m \\
\infty \\
0 \\
\sim \\
0 \\
0\end{array}$ & $\begin{array}{l}1 \\
\infty \\
0 \\
\sim \\
0\end{array}$ & $\begin{array}{l}\mathscr{D} \\
\infty \\
\stackrel{0}{二} \\
\stackrel{0}{0}\end{array}$ & 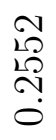 & 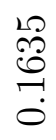 & 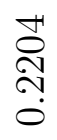 & 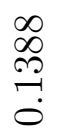 & $\begin{array}{l}0 \\
\text { in } \\
\stackrel{1}{N} \\
\stackrel{0}{0}\end{array}$ & 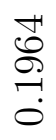 & $\begin{array}{l}\vec{N} \\
\stackrel{N}{N} \\
\stackrel{0}{0}\end{array}$ & $\begin{array}{l}\infty \\
\stackrel{\sim}{\circ} \\
\stackrel{\sim}{0}\end{array}$ & $\begin{array}{l}\stackrel{\infty}{S} \\
\stackrel{+}{+} \\
\stackrel{0}{0}\end{array}$ & 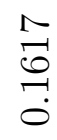 & $\begin{array}{l}+1 \\
\infty \\
\infty \\
\stackrel{-1}{0} \\
\end{array}$ & $\begin{array}{l}\stackrel{N}{N} \\
\text { ָे } \\
\stackrel{0}{0}\end{array}$ & $\begin{array}{l}\stackrel{+}{8} \\
\stackrel{\overbrace{}}{0} \\
\stackrel{0}{0}\end{array}$ & 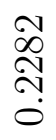 & $\begin{array}{l}\stackrel{0}{\rightrightarrows} \\
\stackrel{\sim}{\sim} \\
\stackrel{0}{0}\end{array}$ \\
\hline $\begin{array}{l}\stackrel{20}{\rightarrow} \\
\| \\
\sim\end{array}$ & $\begin{array}{l}\tilde{D} \\
\stackrel{N}{0} \\
\stackrel{0}{0}\end{array}$ & $\begin{array}{l}\vec{B} \\
\stackrel{B}{10} \\
\overrightarrow{0}\end{array}$ & $\begin{array}{l}\stackrel{?}{1} \\
\stackrel{0}{0} \\
\stackrel{0}{0}\end{array}$ & 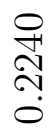 & 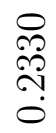 & \begin{tabular}{l}
0 \\
$\infty$ \\
10 \\
$\sim$ \\
\hdashline \\
0
\end{tabular} & $\begin{array}{l}\stackrel{0}{4} \\
\stackrel{\circ}{0} \\
\stackrel{0}{0}\end{array}$ & $\begin{array}{l}\mathscr{R} \\
10 \\
\stackrel{10}{0} \\
0\end{array}$ & $\begin{array}{l}\stackrel{i}{D} \\
\stackrel{N}{N} \\
\stackrel{0}{0}\end{array}$ & 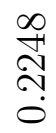 & 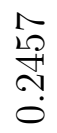 & 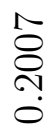 & 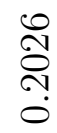 & $\begin{array}{l}8 \\
8 \\
10 \\
\stackrel{10}{0}\end{array}$ & 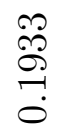 & $\begin{array}{l}\stackrel{8}{8} \\
8 \\
\stackrel{0}{0}\end{array}$ & 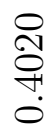 & $\begin{array}{l}\text { N } \\
\stackrel{N}{N} \\
\stackrel{0}{0}\end{array}$ \\
\hline $\begin{array}{c}\stackrel{P}{1} \\
\| \\
\sigma\end{array}$ & $\begin{array}{l}\vec{H} \\
\stackrel{0}{0} \\
\stackrel{0}{0} \\
\dot{0}\end{array}$ & $\begin{array}{l}\stackrel{\vartheta}{0} \\
\stackrel{N}{+} \\
\stackrel{0}{0}\end{array}$ & $\begin{array}{l}\vec{\sigma} \\
0 \\
0 \\
0\end{array}$ & $\begin{array}{l}\mathscr{S} \\
\infty \\
\stackrel{1}{0}\end{array}$ & $\begin{array}{l}\vec{\theta} \\
0 \\
\overrightarrow{0} \\
0\end{array}$ & $\begin{array}{l}10 \\
\infty \\
0 \\
ٌ \\
0\end{array}$ & $\begin{array}{l}\stackrel{+}{I} \\
\text { ב- } \\
0\end{array}$ & 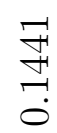 & 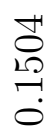 & $\begin{array}{l}\stackrel{2}{2} \\
\stackrel{m}{0} \\
0\end{array}$ & $\begin{array}{l}\infty \\
\infty \\
\beth \\
\stackrel{0}{0}\end{array}$ & 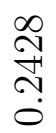 & $\begin{array}{l}\vec{\rho} \\
\overrightarrow{0} \\
\dot{0}\end{array}$ & $\begin{array}{l}\overrightarrow{+} \\
\stackrel{0}{0} \\
\stackrel{-}{0}\end{array}$ & $\begin{array}{l}\vec{H} \\
\overrightarrow{0} \\
\overrightarrow{0}\end{array}$ & $\begin{array}{l}\stackrel{2}{0} \\
\stackrel{0}{0} \\
\stackrel{0}{0}\end{array}$ & $\begin{array}{l}\stackrel{0}{\infty} \\
\stackrel{0}{0} \\
\stackrel{0}{0}\end{array}$ & 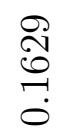 \\
\hline 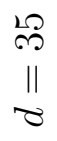 & $\begin{array}{l}\stackrel{\infty}{N} \\
\stackrel{N}{+} \\
\stackrel{0}{0}\end{array}$ & $\begin{array}{l}\text { م⿱ } \\
\text { î } \\
\text { in } \\
0\end{array}$ & $\begin{array}{l}8 \\
\stackrel{0}{0} \\
\stackrel{9}{\rightarrow} \\
\stackrel{0}{0}\end{array}$ & $\begin{array}{l}\stackrel{8}{8} \\
\stackrel{0}{0} \\
\ddot{0}\end{array}$ & $\begin{array}{l}\sqrt{20} \\
\stackrel{2}{二} \\
\stackrel{0}{0}\end{array}$ & $\begin{array}{l}\stackrel{2}{N} \\
\stackrel{N}{0} \\
\stackrel{0}{0}\end{array}$ & $\begin{array}{l}\stackrel{0}{0} \\
\beth \\
\breve{~}\end{array}$ & $\begin{array}{l}\vec{\infty} \\
\stackrel{\infty}{二} \\
\stackrel{0}{0}\end{array}$ & $\begin{array}{l}2 \\
\infty \\
20 \\
\stackrel{10}{0}\end{array}$ & $\begin{array}{l}\stackrel{\Omega}{\Omega} \\
\stackrel{\sim}{=}\end{array}$ & $\begin{array}{l}\stackrel{N}{\sim} \\
\stackrel{\sim}{\sim}\end{array}$ & $\begin{array}{l}\stackrel{H}{+} \\
\stackrel{+}{0}\end{array}$ & 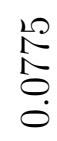 & $\begin{array}{l}\stackrel{\Omega}{\mathbb{O}} \\
\stackrel{1}{0} \\
0\end{array}$ & $\begin{array}{l}\stackrel{2}{0} \\
\stackrel{0}{0} \\
0 \\
0\end{array}$ & $\begin{array}{l}\vec{b} \\
\stackrel{1}{0} \\
0 \\
0\end{array}$ & $\begin{array}{l}\stackrel{0}{\infty} \\
\infty \\
\stackrel{1}{0} \\
\stackrel{0}{0}\end{array}$ & $\begin{array}{l}\infty \\
\stackrel{\infty}{\beth} \\
\end{array}$ \\
\hline $\begin{array}{l}\text { o } \\
\| \\
\sim \\
\sigma\end{array}$ & $\begin{array}{l}\frac{30}{10} \\
\stackrel{2}{+} \\
\dot{0}\end{array}$ & $\begin{array}{l}\stackrel{1}{\text { f }} \\
\stackrel{0}{0} \\
\dot{0}\end{array}$ & 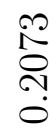 & $\begin{array}{l}\stackrel{1}{\vec{f}} \\
\infty \\
\stackrel{1}{0}\end{array}$ & 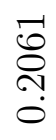 & $\begin{array}{l}\infty \\
\stackrel{+}{\circ} \\
\stackrel{0}{0} \\
\dot{0}\end{array}$ & $\begin{array}{l}\stackrel{2}{O} \\
\stackrel{\leftrightarrow}{+} \\
\stackrel{0}{0}\end{array}$ & $\begin{array}{l}\text { F } \\
\text { 今 } \\
0 \\
0\end{array}$ & $\begin{array}{l}0 \\
20 \\
0 \\
0 \\
0\end{array}$ & $\begin{array}{l}\stackrel{L}{O} \\
\stackrel{0}{0} \\
0\end{array}$ & $\begin{array}{l}\overrightarrow{0} \\
\stackrel{0}{0} \\
0 \\
0\end{array}$ & $\begin{array}{l}\stackrel{10}{\tilde{D}} \\
\stackrel{\sim}{0} \\
\stackrel{0}{0}\end{array}$ & 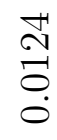 & $\begin{array}{l}\stackrel{\Omega}{0} \\
\stackrel{0}{0} \\
\stackrel{0}{0}\end{array}$ & $\underset{\stackrel{+}{+}}{\stackrel{\vec{\sigma}}{+}}$ & $\begin{array}{l}\stackrel{\infty}{N} \\
\stackrel{N}{0} \\
\stackrel{0}{0}\end{array}$ & 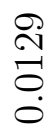 & 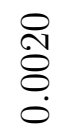 \\
\hline $\begin{array}{l}\stackrel{L}{N} \\
\| \\
\stackrel{\sigma}{\sigma}\end{array}$ & $\begin{array}{l}10 \\
0 \\
0 \\
0 \\
0\end{array}$ & $\begin{array}{l}8 \\
\stackrel{8}{10} \\
\stackrel{+}{0} \\
0\end{array}$ & $\begin{array}{l}\overrightarrow{0} \\
\text { ID } \\
0 \\
0\end{array}$ & $\begin{array}{l}\stackrel{P}{\sim} \\
\stackrel{+}{+} \\
\stackrel{0}{\circ}\end{array}$ & $\begin{array}{l}\infty \\
10 \\
\infty \\
N \\
0 \\
0\end{array}$ & $\begin{array}{l}1 \\
10 \\
\infty \\
0 \\
0\end{array}$ & $\begin{array}{l}\stackrel{0}{1} \\
\stackrel{2}{8} \\
\dot{0}\end{array}$ & $\begin{array}{l}\vec{n} \\
\vec{\sigma}\end{array}$ & $\begin{array}{l}0 \\
\stackrel{1}{1} \\
0 \\
0 \\
0\end{array}$ & 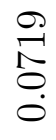 & $\begin{array}{l}\infty \\
\stackrel{\infty}{0} \\
\stackrel{0}{0} \\
\stackrel{0}{0}\end{array}$ & $\begin{array}{l}\stackrel{N}{1} \\
\mathscr{0} \\
0 \\
0\end{array}$ & $\begin{array}{l}\stackrel{9}{+} \\
\stackrel{8}{0} \\
\stackrel{0}{0}\end{array}$ & $\begin{array}{l}\infty \\
\stackrel{P}{1} \\
0 \\
0 \\
0\end{array}$ & $\begin{array}{l}\vec{\infty} \\
\stackrel{+}{0} \\
\dot{0}\end{array}$ & $\begin{array}{l}\stackrel{R}{1} \\
\stackrel{1}{0} \\
\dot{0}\end{array}$ & $\begin{array}{l}10 \\
20 \\
20 \\
0\end{array}$ & $\begin{array}{l}\stackrel{\mathscr{m}}{i \rho}+\underset{0}{0} \\
\stackrel{0}{0}\end{array}$ \\
\hline 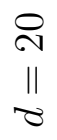 & $\begin{array}{l}\sigma \\
\infty \\
\infty \\
0 \\
\dot{0}\end{array}$ & 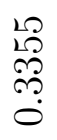 & $\begin{array}{l}0 \\
10 \\
0 \\
0 \\
0 \\
0\end{array}$ & $\begin{array}{l}\text { N } \\
\text { on } \\
\stackrel{2}{0} \\
\stackrel{0}{2}\end{array}$ & 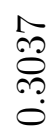 & $\begin{array}{l}\stackrel{\sim}{\leftrightarrow} \\
\stackrel{+}{\circ}\end{array}$ & $\begin{array}{l}0 \\
10 \\
10 \\
0 \\
0 \\
0\end{array}$ & 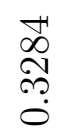 & 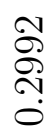 & $\frac{\mathscr{\sigma}}{\stackrel{\sigma}{\vec{\rho}}}$ & 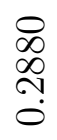 & $\begin{array}{l}\stackrel{N}{N} \\
\stackrel{2}{2} \\
\stackrel{0}{0}\end{array}$ & $\begin{array}{l}\vec{H} \\
\stackrel{2}{2} \\
\stackrel{2}{0}\end{array}$ & $\begin{array}{l}\stackrel{\circ}{0} \\
\stackrel{1}{N} \\
\stackrel{0}{0}\end{array}$ & $\begin{array}{l}10 \\
\infty \\
\dddot{N} \\
\stackrel{0}{0} \\
0\end{array}$ & \begin{tabular}{l}
$\infty$ \\
0 \\
\multirow{N}{N}{} \\
$\stackrel{0}{0}$
\end{tabular} & 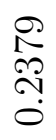 & 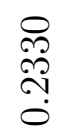 \\
\hline$\stackrel{20}{\underset{\nu}{\|}}$ & $\begin{array}{l}\stackrel{0}{\infty} \\
\stackrel{0}{0} \\
\dot{0}\end{array}$ & 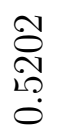 & $\begin{array}{l}\stackrel{H}{0} \\
\stackrel{H}{0} \\
0\end{array}$ & $\begin{array}{l}\mathscr{1} \\
0 \\
10 \\
0 \\
0\end{array}$ & $\begin{array}{l}0 \\
80 \\
10 \\
0 \\
0\end{array}$ & $\begin{array}{l}\stackrel{\rho}{N} \\
\stackrel{\rho}{+} \\
\stackrel{0}{0}\end{array}$ & $\begin{array}{l}0 \\
\mathscr{0} \\
\infty \\
1 ? \\
0\end{array}$ & $\begin{array}{l}\vec{F} \\
\overrightarrow{0} \\
0 \\
0\end{array}$ & $\begin{array}{l}\mathscr{2} \\
0 \\
0 \\
0 \\
0\end{array}$ & $\begin{array}{l}\infty \\
\text { O } \\
\text { i? } \\
0\end{array}$ & $\begin{array}{l}\vec{b} \\
\stackrel{\rho}{+} \\
\dot{0}\end{array}$ & $\begin{array}{l}\vec{\infty} \\
\infty \\
+ \\
+ \\
0\end{array}$ & $\begin{array}{l}\stackrel{\sim}{7} \\
\stackrel{s}{+} \\
\stackrel{\circ}{0}\end{array}$ & $\begin{array}{l}\stackrel{10}{H} \\
\stackrel{6}{7} \\
0\end{array}$ & $\begin{array}{l}\stackrel{N}{N} \\
\stackrel{N}{P} \\
\stackrel{0}{0}\end{array}$ & $\begin{array}{l}\mathscr{\mathscr { A }} \\
\stackrel{\sim}{+} \\
\dot{0}\end{array}$ & $\begin{array}{l}8 \\
\stackrel{9}{7} \\
\stackrel{4}{0}\end{array}$ & 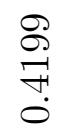 \\
\hline $\begin{array}{l}\stackrel{0}{-1} \\
\| \\
\tau\end{array}$ & 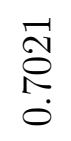 & $\begin{array}{l}1 \\
8 \\
\stackrel{+}{+} \\
\stackrel{0}{0}\end{array}$ & $\begin{array}{l}10 \\
10 \\
10 \\
0 \\
0\end{array}$ & $\begin{array}{l}10 \\
\stackrel{10}{0} \\
\stackrel{2}{P} \\
\stackrel{0}{0}\end{array}$ & $\begin{array}{l}10 \\
0 \\
0 \\
0 \\
0\end{array}$ & $\begin{array}{l}0 \\
\infty \\
\infty \\
0 \\
0\end{array}$ & $\begin{array}{l}\mathscr{\theta} \\
0 \\
+ \\
\dot{0}\end{array}$ & \begin{tabular}{l}
0 \\
\multirow{1}{*}{} \\
$1+2$ \\
0
\end{tabular} & $\begin{array}{l}m \\
\vec{\sigma} \\
\stackrel{0}{0} \\
0\end{array}$ & $\begin{array}{l}\infty \\
\stackrel{\infty}{1} \\
0 \\
0 \\
0\end{array}$ & 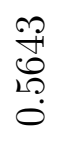 & $\begin{array}{l}\text { i⿱ } \\
\stackrel{10}{10} \\
0\end{array}$ & $\begin{array}{l}\stackrel{o}{0} \\
\stackrel{4}{1 !} \\
0\end{array}$ & $\begin{array}{l}\text { ̊ } \\
\text { N } \\
\text { î. } \\
0\end{array}$ & 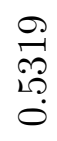 & $\begin{array}{l}10 \\
\text { in } \\
\text { in } \\
0 \\
0\end{array}$ & 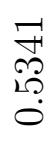 & $\begin{array}{l}1 \\
\text { No } \\
\text { î. } \\
0\end{array}$ \\
\hline$\underset{\sigma}{-\vec{H}}$ & $\begin{array}{l}\overrightarrow{\mathscr{O}} \\
\infty \\
20 \\
0 \\
0\end{array}$ & $\begin{array}{l}\infty \\
\stackrel{0}{0} \\
\mathscr{0} \\
0 \\
0\end{array}$ & $\begin{array}{l}\infty \\
0 \\
0 \\
0 \\
0 \\
0\end{array}$ & $\begin{array}{l}\infty \\
\mathscr{0} \\
\mathscr{0} \\
\dot{0} \\
\dot{0}\end{array}$ & $\begin{array}{l}\infty \\
0 \\
0 \\
0 \\
0 \\
0\end{array}$ & $\begin{array}{l}\infty \\
0 \\
0 \\
0 \\
0 \\
0\end{array}$ & $\begin{array}{l}\infty \\
\mathscr{0} \\
0 \\
0 \\
0\end{array}$ & $\begin{array}{l}\infty \\
0 \\
0 \\
0 \\
0 \\
0\end{array}$ & $\begin{array}{l}\infty \\
0 \\
0 \\
0 \\
0 \\
0\end{array}$ & $\begin{array}{l}\infty \\
0 \\
0 \\
0 \\
0 \\
0\end{array}$ & $\begin{array}{l}\infty \\
\mathscr{8} \\
0 \\
0 \\
0\end{array}$ & $\begin{array}{l}\infty \\
\mathscr{0} \\
0 \\
0 \\
0\end{array}$ & $\begin{array}{l}\infty \\
0 \\
0 \\
0 \\
0 \\
0\end{array}$ & $\begin{array}{l}\infty \\
0 \\
0 \\
0 \\
0 \\
0\end{array}$ & $\begin{array}{l}\infty \\
0 \\
0 \\
0 \\
0 \\
0\end{array}$ & $\begin{array}{l}\infty \\
0 \\
0 \\
0 \\
0 \\
0\end{array}$ & $\begin{array}{l}\infty \\
0 \\
0 \\
0 \\
0 \\
0\end{array}$ & $\begin{array}{l}\infty \\
0 \\
\mathscr{0} \\
0 \\
0\end{array}$ \\
\hline 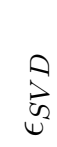 & $\begin{array}{l}\vec{d} \\
\stackrel{d}{8} \\
\end{array}$ & 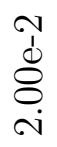 & $\begin{array}{l}N \\
d \\
\delta \\
\\
\end{array}$ & $\begin{array}{c}m \\
\dot{d} \\
\delta \\
\delta \\
0\end{array}$ & $\begin{array}{r}\infty \\
\dot{d} \\
\stackrel{8}{0} \\
\dot{\forall}\end{array}$ & 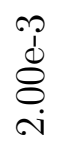 & 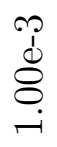 & 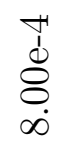 & $\begin{array}{l}+1 \\
d \\
\delta \\
0 \\
0\end{array}$ & $\begin{array}{r}\overrightarrow{1} \\
\stackrel{\delta}{\delta} \\
\delta \\
0\end{array}$ & $\begin{array}{l}\stackrel{+}{d} \\
\stackrel{d}{8} \\
\stackrel{+}{8}\end{array}$ & $\begin{array}{l}\overrightarrow{1} \\
d \\
\stackrel{d}{\delta} \\
\dot{0}\end{array}$ & 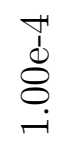 & $\begin{array}{c}1 \\
\dot{d} \\
\stackrel{\delta}{0} \\
\dot{\infty}\end{array}$ & $\begin{array}{c}10 \\
0 \\
\delta \\
\delta \\
0 \\
0\end{array}$ & 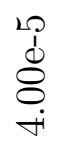 & 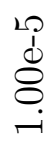 & $\begin{array}{l}\infty \\
1 \\
\delta \\
\delta \\
\end{array}$ \\
\hline
\end{tabular}




\section{References}

[1] Machowski, J. and Bialek, J. and Bumby, J., Power system dynamics: stability and control, John Wiley \& Sons, 2011.

[2] Schmid, P., Dynamic mode decomposition of numerical and experimental data, Journal of Fluid Mechanics 656 (2010) 5-28, ISSN 0022-1120, URL http://search.proquest.com/docview/753672207/.

[3] Susuki, Y. and Mezic, I., Nonlinear Koopman Modes and Power System Stability Assessment Without Models, Power Systems, IEEE Transactions on 29 (2) (2014) 899-907, ISSN 0885-8950.

[4] Barocio, E. and Pal, B. C. and Thornhill, N. F. and Messina, A. R., A Dynamic Mode Decomposition Framework for Global Power System Oscillation Analysis, Power Systems, IEEE Transactions on 30 (6) (2015) 2902-2912, ISSN 0885-8950.

[5] Ramos, J. J. and Kutz, J. N., Dynamic Mode Decomposition and Sparse Measurements for Characterization and Monitoring of Power System Disturbances, arXiv preprint arXiv:1906.03544 .

[6] Koopman, B. O., Hamiltonian systems and transformation in Hilbert space, Proceedings of the National Academy of Sciences of the United States of America 17 (5) (1931) 315.

[7] Mezić, I., Spectral properties of dynamical systems, model reduction and decompositions, Nonlinear Dynamics 41 (1-3) (2005) 309-325. 
[8] Le Clainche, S. and Vega, J. M., Higher order dynamic mode decomposition, SIAM Journal on Applied Dynamical Systems 16 (2) (2017) 882-925.

[9] Takens, F., Detecting strange attractors in turbulence, in: Dynamical Systems and Turbulence, Warwick 1980, Lecture Notes in Mathematics, Springer Berlin Heidelberg, Berlin, Heidelberg, ISBN 3540111719, 366381, 2006.

[10] Strang, G., Introduction to linear algebra, 9780980232776, WellesleyCambridge Press, Wellesley, 2016.

[11] Le Clainche, S. and Vega, J. M., Higher order dynamic mode decomposition to identify and extrapolate flow patterns, Physics of Fluids 29 (8) (2017) 084102.

[12] Le Clainche, S. and Vega, J. M., Analyzing nonlinear dynamics via datadriven dynamic mode decomposition-like methods, Complexity 2018.

[13] Le Clainche, S. and Ferrer, E., A reduced order model to predict transient flows around straight bladed vertical axis wind turbines, Energies 11 (3) (2018) 566.

[14] Vega, J. M. and Le Clainche, S., Higher order dynamic mode decomposition and its applications, Academic Press, London, United Kingdom, ISBN 9780128227664, 2021.

[15] Alassaf, A. and Fan, L., Randomized Dynamic Mode Decomposition for Oscillation Modal Analysis, IEEE transactions on power systems 36 (2) (2021) 1399-1408, ISSN 0885-8950. 
[16] Erichson, N. B. and Mathelin, L. and Kutz, J. N. and Brunton, S. L., Randomized dynamic mode decomposition, SIAM journal on applied dynamical systems 18 (4) (2019) 1867-1891, ISSN 1536-0040.

[17] Dawson, S. T. M. and Hemati, M. S. and Williams, M. O. and Rowley, C. W., Characterizing and correcting for the effect of sensor noise in the dynamic mode decomposition, Experiments in Fluids 57 (3) (2016) 42.

[18] Cheng, C. and Ding, J. and Zhang, Y., A Koopman operator approach for machinery health monitoring and prediction with noisy and lowdimensional industrial time series, Neurocomputing .

[19] Kou, J. and Zhang, W., An improved criterion to select dominant modes from dynamic mode decomposition, European Journal of MechanicsB/Fluids 62 (2017) 109-129.

[20] Kutz, J. N. and Brunton, S. L. and Brunton, B. W. and Proctor, J. L., Dynamic mode decomposition: data-driven modeling of complex systems, Other Titles in Applied Mathematics, SIAM, ISBN 9781611974508, 2016.

[21] Tu, J. H. and Rowley, C. W. and Luchtenburg, D. M. and Brunton, S. L. and Kutz, J. N., On dynamic mode decomposition: theory and applications, arXiv preprint arXiv:1312.0041 .

[22] Hemati, M. S. and Rowley, C. W. and Deem, E. A. and Cattafesta, L. N., De-biasing the dynamic mode decomposition for applied Koopman spectral analysis of noisy datasets, Theoretical and Computatio- 
nal Fluid Dynamics, https://doi.org/10.1007/s00162-017-0432-2 31 (4) (2017) 349-368.

[23] Hirsh, S. M. and Harris, K. D. and Kutz, J. N. and Brunton, B. W., Centering Data Improves the Dynamic Mode Decomposition, arXiv preprint arXiv:1906.05973 .

[24] Hua, J.-C. and Noorian, F. and Moss, D. and Leong, P. H. W. and Gunaratne, G. H., High-dimensional time series prediction using kernelbased Koopman mode regression, Nonlinear Dynamics 90 (3) (2017) $1785-1806$.

[25] UN-SCAN-IT, Graph Digitizing Software, Version 6.2, Silk Scientific Corporation, 2018.

[26] Le Clainche, Soledad and Vega, José, "Book Higher Order Dynamic Mode Decomposition and its applications: MATLAB codes", Mendeley Data, V1, doi: 10.17632/z8ks4f5vy5.1, (2020).

[27] Australian Energy Market Operator, Final Report - Queensland and South Australia system separation on 25 August 2018, Tech. Rep., An operating incident report for the National Electricity Market, January 2019 .

[28] Higham, N., higham/matlab-guide-3ed, GitHub, URL https:// github.com/higham/matlab-guide-3ed, (2021). Retrieved August 4, 2021.

[29] MATLAB, version 9.10.0.1602886 (R2021a), The MathWorks Inc., Natick, Massachusetts, 2021. 
[30] Ben-Hur, E., https://stackoverflow.com/questions/44729085/how-tochange-the-font-size-of-axes-and-line-weight-in-boxplot, 2017.

[31] National Grid ESO, Technical Report on the Events of 9 August 2019, 6 September 2019.

[32] National Grid ESO, URL https://www.nationalgrideso. com/balancing-services/frequency-response-services/ historic-frequency-data, 2019.

[33] Maas, G. A. and Bial, M. and Fijalkowski, J., Final Report - System Disturbance on 4 November 2006, Union for the Coordination of Transmission of Electricity in Europe, Tech. Report . 